\title{
Reinforcement problems for variational inequalities on fractal sets
}

\author{
Raffaela Capitanelli $^{1}$ - Maria Agostina Vivaldi ${ }^{1}$
}

Received: 7 November 2014 / Accepted: 5 June 2015 / Published online: 8 July 2015

(C) Springer-Verlag Berlin Heidelberg 2015

\begin{abstract}
The aim of this paper is to study reinforcement problems for variational inequalities of the obstacle type on fractal sets.
\end{abstract}

Mathematics Subject Classification $28 \mathrm{~A} 80 \cdot 35 \mathrm{~J} 20 \cdot 49 \mathrm{~A} 29$

\section{Introduction}

The aim of this paper is to study reinforcement problems for variational inequalities on fractal sets.

The obstacle problem is a classic motivating example in the mathematical study of variational inequalities and free boundary problems. The problem entails finding the equilibrium position of an elastic membrane whose boundary is held fixed, and which is constrained to lie above (or under) a given obstacle. It is closely related to the study of minimal surfaces as well as to the capacity of a set in potential theory. Applications include the study of elastoplasticity, optimal control, fluid filtration in porous media, constrained heating, and financial mathematics (see, for example, [8]).

The theory of variational inequalities starts from the paper [31] of Stampacchia: it was in fact in this paper that the name "variational inequalities" was introduced. The theory was subsequently further developed in the paper [22], in the book [20] and, later, in many papers and books (we refer to [8,32], and the references therein).

Communicated by L. Caffarelli.

Raffaela Capitanelli

raffaela.capitanelli@uniroma1.it

Maria Agostina Vivaldi

maria.vivaldi@sbai.uniroma1.it

1 Dipartimento di Scienze di Base e Applicate per l'Ingegneria, "Sapienza” Università di Roma, Via A. Scarpa 1600161 Rome, Italy 
In this paper, we state the existence, uniqueness and approximation results for variational solutions of obstacle problems on domains with a fractal boundary. The fractal solution can be approximated by solutions of obstacle problems related to the same operator in polygonal domains whose boundaries develop, at the limit, fractal geometry (extending the results of [9] and in [10]).

However, in this paper we prefer to adopt the approach of reinforcement by means of thin insulating layers according to the approach adopted in the celebrated paper by Brezis, Caffarelli and Friedman ([3]). We mention for related results the contributions [1,2,5-7,21, 30]. All these papers concern smooth domains (at least Lipschitz) and use tools and methods that can not be extended to domains with a fractal boundary.

The homogenization theory for domains with a fractal boundary have been developed in [26-28], and [29] for highly conductive layers and in [11,13], and [14] for insulating layers. We also wish to mention $[4,15]$, where the reinforcement has a different structure.

A peculiar aspect of insulating layers is the loss of coerciveness of the energy functionals; moreover, in the case of fractals, this aspect is combined with the tricky geometry of the fibers. We overcome these difficulties by using some delicate tools such as extension theorems for $(\epsilon, \delta)$ domains established by Jones (see [17]), sharp quantitative trace results (on polygonal curves) in terms of the increasing numbers of sides (see [12]), and establishing Poincaré type estimates adapted to the geometry (see Theorems 3.2, 7.1, 7.4). We study obstacle problems for both coercive energy forms and semi-coercive energy forms.

We wish to point out that Theorems 5.1 and 5.2 improve the results of [11] and [13] insofar as the hypotheses are weaker and the convergence of the approximating solutions is stated in a more precise way (see Remark 5.1). Theorems 5.3 and 7.5 concern the semicoercive case and the relative results are completely new to our knowledge. Moreover, simple examples in Sect. 6 show that our results are sharp.

We note that the fractal setting gives rise to a peculiar phenomenon. Owing to their tricky geometry, the reinforced domains have to be constructed starting from suitable inner polygonal domains $\Omega^{n}$. Hence the forms $a_{n}$ [see (3.3) and (3.5)] vanish in a part of the fractal domain (in $\Omega \backslash \Omega^{n}$ ) when the thickness of the layers goes to zero and, for fixed $n$, the forms $a_{n}$ degenerate at the vertices of the polygonal curves $K_{j, \alpha}^{n}, j=1,2,3$. Consequently, the reinforced solutions have gradients that are not uniformly bounded in $L^{2}$-norms (in $\Omega \backslash \Omega^{n}$ ), which is in contrast to the strong convergence of the gradients (in $L^{2}$ ) established in [3]; compare Theorem 5.3 with Theorem 9.3 in [3] (see Remark 5.4).

The plan of the paper is as follows. In Sect. 2, we introduce the fractal domains, we set up some obstacle problems and we state existence and uniqueness results of variational solutions. Section 3 is devoted to constructing suitable reinforced problems and to proving existence and uniqueness of the related solutions. In Sect. 4, we state Mosco-convergence of the related functionals. Section 5 concerns the asymptotic results. In Sect. 6, we comment on our results by discussing some simple examples. Finally, in Sect. 7 we deal with interior reinforcement.

\section{Obstacle problems on fractal domains}

First, we introduce the fractal domains in which we consider the obstacle problems. We recall the definition of the Koch curve with endpoints $A=(0,0)$, and $B=(1,0)$. We consider the family $\Psi^{\alpha}=\left\{\psi_{1}^{\alpha}, \ldots, \psi_{4}^{\alpha}\right\}$ of contractive similitudes $\psi_{i}^{\alpha}: \mathbb{C} \rightarrow \mathbb{C}, i=1, \ldots, 4$, with contraction factor $\alpha^{-1}, 2<\alpha<4$, 


$$
\begin{aligned}
& \psi_{1}^{\alpha}(z)=\frac{z}{\alpha}, \quad \psi_{2}^{\alpha}(z)=\frac{z}{\alpha} e^{i \theta(\alpha)}+\frac{1}{\alpha} \\
& \psi_{3}^{\alpha}(z)=\frac{z}{\alpha} e^{-i \theta(\alpha)}+\frac{1}{2}+i \sqrt{\frac{1}{\alpha}-\frac{1}{4}}, \quad \psi_{4}^{\alpha}(z)=\frac{z-1}{\alpha}+1,
\end{aligned}
$$

where $\theta(\alpha)=\arcsin \left(\frac{\sqrt{\alpha(4-\alpha)}}{2}\right)$. According to the general theory of self-similar fractals (see $[16,19])$, there exists a unique closed bounded set $K_{\alpha}$ which is invariant with respect to $\Psi^{\alpha}$, that is,

$$
K_{\alpha}=\cup_{i=1}^{4} \psi_{i}^{\alpha}\left(K_{\alpha}\right) .
$$

We recall that $K_{\alpha}$ supports a unique self-similar Borel measure $\mu_{\alpha}$, which is equivalent to the $d_{f}$-dimensional Hausdorff measure where $d_{f}=\frac{\log 4}{\log \alpha}$. Let $K^{0}$ be the line segment of unit length that has as endpoints $A=(0,0)$ and $B=(1,0)$. We set, for each $n$ in $\mathbb{N}$,

$$
K_{\alpha}^{1}=\bigcup_{i=1}^{4} \psi_{i}^{\alpha}\left(K^{0}\right), \quad K_{\alpha}^{2}=\bigcup_{i=1}^{4} \psi_{i}^{\alpha}\left(K_{\alpha}^{1}\right), \ldots, K_{\alpha}^{n+1}=\bigcup_{i=1}^{4} \psi_{i}^{\alpha}\left(K_{\alpha}^{n}\right)
$$

$K_{\alpha}^{n}$ is the so-called $n$th pre-fractal curve. Moreover, the iterates $K_{\alpha}^{n}$ converge to the selfsimilar set $K_{\alpha}$ in the Hausdorff metric, when $n$ tends to infinity. Let $\Omega^{0}$ be the triangle with vertices $A=(0,0), B=(1,0)$, and $C=\left(\frac{1}{2},-\frac{\sqrt{3}}{2}\right)$. We construct, on the side with endpoints $A$ and $B$, the pre-fractal Koch curve defined above, which will be denoted by $K_{1, \alpha}^{n}$ and the Koch curve defined above, which will be denoted by $K_{1, \alpha}$. Similarly, we construct on the other sides the analogous pre-fractal Koch curves (the Koch curves) denoting by $K_{2, \alpha}^{n}$ and $K_{3, \alpha}^{n}$ (by $K_{2, \alpha}$ and $K_{3, \alpha}$ ) the curves with endpoints $B$ and $C$, and $C$ and $A$, respectively. We denote by $\Omega_{\alpha}^{n}$ the pre-fractal domain that is the set bounded by the pre-fractal Koch curves $K_{j, \alpha}^{n}, j=1,2,3$. Moreover, we denote by $\Omega_{\alpha}$ the snowflake that is the set bounded by the Koch curves $K_{j, \alpha}, j=1,2,3$. From now on, we omit $\alpha$ when it does not give rise to misunderstanding, by writing simply $\Omega$ instead of $\Omega_{\alpha}$ and similar expressions.

In the following theorems, we state the existence and uniqueness of the variational solution of obstacle problems on the domain $\Omega$. We consider the following bilinear forms

$$
a_{\infty}(u, v):=\int_{\Omega} \sum_{i, j=1}^{2} a_{i j} \frac{\partial u}{\partial x_{i}} \frac{\partial v}{\partial x_{j}} d x d y+\delta_{0} \int_{\Omega} u v d x d y
$$

with domain $H_{0}^{1}(\Omega)$ and

$$
a_{c_{0}}(u, v):=\int_{\Omega} \sum_{i, j=1}^{2} a_{i j} \frac{\partial u}{\partial x_{i}} \frac{\partial v}{\partial x_{j}} d x d y+\delta_{0} \int_{\Omega} u v d x d y+c_{0} \int_{\partial \Omega} u v d \mu
$$

with domain $H^{1}(\Omega)$. In the last integral, $\mu$ is the measure on $\partial \Omega$ that coincides, on each $K_{j}$ $j=1,2,3$, with the Hausdorff measure defined in this section previously and $u$ and $v$ denote the traces of the functions $u$ and $v$ on the boundary of $\Omega$. Here

$$
\delta_{0} \geq 0
$$

and the coefficients $a_{i j}$ with $1 \leq i, j \leq 2$ satisfy

$$
a_{i j}=a_{j i} \quad \forall i, j, \text { and } \lambda|\xi|^{2} \leq \sum_{i, j=1}^{2} a_{i j} \xi_{i} \xi_{j} \leq \Lambda|\xi|^{2}
$$


for $\Lambda \geq \lambda>0$. Furthermore, we assume the following condition

$$
\mathcal{K}_{0}=\left\{u \in H_{0}^{1}(\Omega), u \geq \varphi_{1}\right\}, \varphi_{1} \in C^{1}(\bar{\Omega}), \quad \varphi_{1} \leq 0 \quad \text { on } \partial \Omega .
$$

We state the following result. Since the proof is similar to the proof of Theorem 2.2, we skip it. From now on, when it does not give rise to misunderstanding, we denote by $C$ possibly different constants.

Theorem 2.1 Let us assume (2.5)-(2.7). Then, for any $f \in L^{2}(\Omega)$, there exists one and only one solution $u$ to the following problem

$$
\left\{\begin{array}{l}
\text { find } u \in \mathcal{K}_{0} \text { such that } \\
a_{\infty}(u, v-u) \geq \int_{\Omega} f(v-u) d x d y \quad \forall v \in \mathcal{K}_{0}
\end{array}\right.
$$

where $a_{\infty}(\cdot, \cdot)$ is defined in (2.3). Moreover, $u$ is the only function that realizes the minimum of the energy functional

$$
\min _{v \in \mathcal{K}_{0}}\left\{a_{\infty}(v, v)-2 \int_{\Omega} f v d x d y\right\}
$$

and

$$
\|u\|_{H^{1}(\Omega)} \leq C\left(\|f\|_{L^{2}(\Omega)}+\left\|\varphi_{1}\right\|_{C^{1}(\bar{\Omega})} .\right.
$$

Remark 2.1 A similar result holds for the two obstacle problems where

$$
\varphi_{h} \in C^{1}(\bar{\Omega}), \quad h=1,2 \quad \varphi_{1} \leq \varphi_{2}, \quad \varphi_{1} \leq 0 \leq \varphi_{2} \quad \text { on } \partial \Omega
$$

and

$$
\mathcal{K}_{0}^{*}=\left\{u \in H_{0}^{1}(\Omega), \quad \varphi_{1} \leq u \leq \varphi_{2}\right\} .
$$

Moreover, the following estimates hold

$$
\begin{aligned}
\|u\|_{H^{1}(\Omega)} & \leq C\left(\|f\|_{L^{2}(\Omega)}+\min \left(\left\|\varphi_{1}\right\|_{C^{1}(\bar{\Omega})},\left\|\varphi_{2}\right\|_{C^{1}(\bar{\Omega})}\right)\right) \\
\|u\|_{L^{\infty}(\Omega)} & \leq \max \left(\left\|\varphi_{1}\right\|_{C^{0}(\bar{\Omega})},\left\|\varphi_{2}\right\|_{C^{0}(\bar{\Omega})}\right) .
\end{aligned}
$$

Now we assume that

$$
c_{0} \geq 0, \delta_{0} \geq 0, \quad \text { and } \max \left(c_{0}, \delta_{0}\right)>0 .
$$

Let

$$
\mathcal{K}=\left\{u \in H^{1}(\Omega), \quad u \geq \varphi_{1}\right\}, \quad \varphi_{1} \in C^{1}(\bar{\Omega}) .
$$

Theorem 2.2 Let us assume (2.6), (2.14), (2.15) and $d \in \mathbb{R}$. Then, for any $f \in L^{2}(\Omega)$, there exists one and only one solution $u$ to the following problem

$$
\left\{\begin{array}{l}
\text { find } u \in \mathcal{K} \text { such that } \\
a_{c_{0}}(u, v-u) \geq \int_{\Omega} f(v-u) d x d y+d \int_{\partial \Omega}(v-u) d \mu \quad \forall v \in \mathcal{K}
\end{array}\right.
$$

where $a_{c_{0}}(\cdot, \cdot)$ is defined in (2.4). Moreover, $u$ is the only function that realizes the minimum of the energy functional

$$
\min _{v \in \mathcal{K}}\left\{a_{c_{0}}(v, v)-2 \int_{\Omega} f v d x d y-2 d \int_{\partial \Omega} v d \mu\right\}
$$


and

$$
\|u\|_{H^{1}(\Omega)} \leq C\left(\|f\|_{L^{2}(\Omega)}+|d|+\left\|\varphi_{1}\right\|_{C^{1}(\bar{\Omega})}\right) .
$$

Proof By Trace Theorem of Chapter V in [18] and Extension Theorem in [17], we have that

$$
\left|d \int_{\partial \Omega} v d \mu\right| \leq C|d|\|v\|_{H^{1}(\Omega)}
$$

and

$$
\left|\int_{\Omega} f v d x d y\right| \leq\|f\|_{L^{2}(\Omega)}\|v\|_{L^{2}(\Omega)} .
$$

The bilinear form $a_{c_{0}}(u, v)$ is continuous. Indeed, by using Trace Theorem (see Chapter $\mathrm{V}$ in [18]) again

$$
\left|a_{c_{0}}(u, v)\right| \leq\left(\max \left(\delta_{0}, \Lambda\right)+c_{0} C\right)\|u\|_{H^{1}(\Omega)}\|v\|_{H^{1}(\Omega)} .
$$

Moreover, the form is coercive. In fact, if $\delta_{0}>0$ we obtain trivially

$$
a_{c_{0}}(v, v) \geq \min \left(\delta_{0}, \lambda\right)\|v\|_{H^{1}(\Omega)}^{2} .
$$

Instead, if $\delta_{0}=0$ and $c_{0}>0$ by using generalized Poincaré inequality (see Lemma 3.1.1 in [23]), we obtain that

$$
a_{c_{0}}(v, v) \geq C \min \left(c_{0}, \lambda\right)\|v\|_{H^{1}(\Omega)}^{2} .
$$

Remark 2.2 A similar result holds for the two obstacle problems where

$$
\varphi_{h} \in C^{1}(\bar{\Omega}), \quad h=1,2 \text { and } \varphi_{1} \leq \varphi_{2}
$$

and

$$
\mathcal{K}^{*}=\left\{u \in H^{1}(\Omega), \quad \varphi_{1} \leq u \leq \varphi_{2}\right\} .
$$

Moreover, the following estimates hold

$$
\begin{aligned}
& \|u\|_{H^{1}(\Omega)} \leq C\left(\|f\|_{L^{2}(\Omega)}+|d|+\min \left(\left\|\varphi_{1}\right\|_{C^{1}(\bar{\Omega})},\left\|\varphi_{2}\right\|_{C^{1}(\bar{\Omega})}\right)\right) \\
& \|u\|_{L^{\infty}(\Omega)} \leq \max \left(\left\|\varphi_{1}\right\|_{C^{0}(\bar{\Omega})},\left\|\varphi_{2}\right\|_{C^{0}(\bar{\Omega})}\right) .
\end{aligned}
$$

If $c_{0}=\delta_{0}=0$, we can prove similar results by assuming further conditions on the data $f, d$ and on the convex. For the sake of simplicity, we take

$$
0 \in \mathcal{K}
$$

and

$$
\int_{\Omega} f d x d y+d \int_{\partial \Omega} d \mu<0
$$

Theorem 2.3 Let us assume $d \in \mathbb{R}$, (2.6), (2.15), (2.23), and (2.24). Then, for any $f \in$ $L^{2}(\Omega)$, there exists one and only one solution $u$ to the following problem

$$
\left\{\begin{array}{l}
\text { find } u \in \mathcal{K} \text { such that } \\
a(u, v-u) \geq \int_{\Omega} f(v-u) d x d y+d \int_{\partial \Omega}(v-u) d \mu \quad \forall v \in \mathcal{K}
\end{array}\right.
$$


where $a(u, v)=\int_{\Omega} \sum_{i, j=1}^{2} a_{i j} \frac{\partial u}{\partial x_{i}} \frac{\partial v}{\partial x_{j}} d x d y$. Moreover, $u$ is the only function that realizes the minimum of the energy functional

$$
\min _{v \in \mathcal{K}}\left\{a(v, v)-2 \int_{\Omega} f v d x d y-2 d \int_{\partial \Omega} v d \mu\right\}
$$

and

$$
\|\nabla u\|_{L^{2}(\Omega)} \leq C\left(\|f\|_{L^{2}(\Omega)}+|d|+\left\|\varphi_{1}\right\|_{C^{1}(\bar{\Omega})}\right) .
$$

Proof The existence can be proved as in Theorem 5.1 in [22] (see also Theorem 4.7 in [32]). We show the uniqueness by contradiction. Let $u_{1}$ and $u_{2}$ be two solutions of (2.25). As

$$
a\left(u_{1}, u_{1}-u_{2}\right) \leq \int_{\Omega} f\left(u_{1}-u_{2}\right) d x d y+d \int_{\partial \Omega}\left(u_{1}-u_{2}\right) d \mu
$$

and

$$
a\left(u_{2}, u_{2}-u_{1}\right) \leq \int_{\Omega} f\left(u_{2}-u_{1}\right) d x d y+d \int_{\partial \Omega}\left(u_{2}-u_{1}\right) d \mu
$$

we obtain

$$
\lambda\left\|\nabla\left(u_{2}-u_{1}\right)\right\|_{L^{2}(\Omega)}^{2} \leq a\left(u_{1}-u_{2}, u_{1}-u_{2}\right) \leq 0
$$

and therefore $u_{1}=u_{2}+k_{2}$. From (2.28) we deduce

$$
0=a\left(u_{1}, k_{2}\right) \leq \int_{\Omega} f k_{2} d x d y+d \int_{\partial \Omega} k_{2} d \mu:
$$

then, from (2.24),we obtain that $k_{2} \leq 0$. From (2.29) we deduce

$$
0=a\left(u_{2},-k_{2}\right) \leq-\int_{\Omega} f k_{2} d x d y-d \int_{\partial \Omega} k_{2} d \mu:
$$

then, from (2.24), we obtain that $-k_{2} \leq 0$ : therefore, $k_{2}=0$.

Remark 2.3 Similar results hold for the obstacle problem where

$$
\mathcal{K}^{\#}=\left\{u \in H^{1}(\Omega), \quad u \leq \varphi_{2}\right\}, \quad \varphi_{2} \in C^{1}(\bar{\Omega})
$$

by assuming

$$
0 \in \mathcal{K}^{\#}
$$

and

$$
\int_{\Omega} f d x d y+d \int_{\partial \Omega} d \mu>0
$$

Remark 2.4 Analogous results hold for the two obstacle problems where $\mathcal{K}^{*}$ is defined in (2.20) assuming condition (2.19) and

$$
0 \in \mathcal{K}^{*}
$$

Moreover, the following estimates hold

$$
\begin{aligned}
& \|\nabla u\|_{L^{2}(\Omega)} \leq C\left(\|f\|_{L^{2}(\Omega)}+|d|+\min \left(\left\|\varphi_{1}\right\|_{C^{1}(\bar{\Omega})},\left\|\varphi_{2}\right\|_{C^{1}(\bar{\Omega})}\right)\right) \\
& \|u\|_{L^{\infty}(\Omega)} \leq \max \left(\left\|\varphi_{1}\right\|_{C^{0}(\bar{\Omega})},\left\|\varphi_{2}\right\|_{C^{0}(\bar{\Omega})}\right) .
\end{aligned}
$$




\section{Reinforcement for variational inequalities}

We denote by $\Sigma_{1}^{0}$ the open triangle of vertices $A=(0,0), B=(1,0)$ and $C=(1 / 2, b / 2)$ where $b=\tan \left(\frac{\theta}{2}\right)$. For each integer $n>0$ we denote by

$$
\psi_{i \mid n}=\psi_{i_{1}} \circ \psi_{i_{2}} \circ \cdots \circ \psi_{i_{n}}
$$

the map associated with arbitrary $n$-tuple of indices $i \mid n=\left(i_{1}, i_{2}, \ldots, i_{n}\right) \in\{1, \ldots, 4\}^{n}$. If $n=0$ we define $\psi_{i \mid n}$ to be the identity map in $\mathbb{R}^{2}$. For every set $\mathcal{O} \subseteq \mathbb{R}^{2}$, we define $\mathcal{O}^{i \mid n}=\psi_{i \mid n}(\mathcal{O})$, and, occasionally, we call $i \mid n$ the $n$-address of the set $\mathcal{O}^{i \mid n}$. With this notation, the polygonal curve $K_{\alpha}^{n}$ defined in (2.2) can be written $K_{\alpha}^{n}=\bigcup_{i \mid n} \psi_{i \mid n}\left(K^{0}\right)$. The triangle $\Sigma_{1}^{0}$ satisfies the open set condition with respect to the maps $\Psi$, that is, $\psi_{i \mid n}\left(\Sigma_{1}^{0}\right) \subset \Sigma_{1}^{0}$ for every $i \mid n$ and $\psi_{i \mid n}\left(\Sigma_{1}^{0}\right) \cap \psi_{j \mid n}\left(\Sigma_{1}^{0}\right)=\emptyset$ for every $i|n \neq j| n$ (see, for details, $[16,19]$ ).

For every $n \in \mathbb{N} \cup\{0\}$, we define the fiber $\Sigma_{1, \alpha}^{n}$, of $K_{1, \alpha}^{n}$ to be the (open) set

$$
\Sigma_{1, \alpha}^{n}=\bigcup_{i \mid n} \Sigma_{1, \alpha}^{i \mid n}
$$

where

$$
\Sigma_{1, \alpha}^{i \mid n}=\psi_{i \mid n}\left(\Sigma_{1}^{0}\right) .
$$

We proceed in a similar way in order to construct the fiber $\Sigma_{j, \alpha}^{n},(j=2,3)$, and we define the fiber $\Sigma^{n}$,

$$
\Sigma^{n}=\Sigma_{\alpha}^{n}=\bigcup_{j=1}^{3} \Sigma_{j, \alpha}^{n}=\bigcup_{j=1}^{3} \Sigma_{j}^{n}
$$

and

$$
\widehat{\Omega}^{n}=\widehat{\Omega}_{\alpha}^{n}:=\operatorname{int}\left(\overline{\Omega_{\alpha}^{n}} \bigcup \Sigma_{\alpha}^{n}\right)
$$

We note that

$$
\Omega^{n} \subset \Omega^{n+1} \subset \Omega \subset \widehat{\Omega}^{n+1} \subset \widehat{\Omega}^{n} .
$$

We define a weight $w^{n}$ as follows. Let $P$-for some $i \mid n$-belong to $\partial\left(\Sigma_{1}^{i \mid n}\right) \backslash\left(K^{0}\right)^{i \mid n}$ and let $P^{\perp}$ be the orthogonal projection of $P$ on $\left(K^{0}\right)^{i \mid n}$. If $(x, y)$ belongs to the segment with end-points $P$ and $P^{\perp}$, we set, in our current notation,

$$
w_{1}^{n}(x, y)=\frac{3\left|P-P^{\perp}\right|}{3+b^{2}}
$$

where $\left|P-P^{\perp}\right|$ is the (Euclidean) distance between $P$ and $P^{\perp}$ in $\mathbb{R}^{2}$. We proceed in a similar way to construct the weights $w_{j}^{n}$ on $\Sigma_{j}^{n}(j=2,3)$ and we define $w^{n}$ on $\widehat{\Omega}^{n}$

$$
w^{n}(x, y)= \begin{cases}w_{j}^{n}(x, y) & \text { if }(x, y) \in \Sigma_{j}^{n} \\ 1 & \text { if }(x, y) \in \bar{\Omega}^{n}\end{cases}
$$

Associated with the weight $w^{n}$, we consider the Sobolev spaces $H^{1}\left(\widehat{\Omega}^{n} ; w^{n}\right)$ and $H_{0}^{1}\left(\widehat{\Omega}^{n} ; w^{n}\right)$, defined as the completion of $C^{\infty}\left({\widehat{\Omega^{n}}}^{n}\right)$ and $C_{0}^{\infty}\left(\widehat{\Omega}^{n}\right)$, respectively, in the norm

$$
\|u\|_{H^{1}\left(\widehat{\Omega}^{n} ; w^{n}\right)}=\left\{\int_{\widehat{\Omega}^{n}} u^{2} d x d y+\int_{\widehat{\Omega}^{n}}|\nabla u|^{2} w^{n} d x d y\right\}^{\frac{1}{2}} .
$$


We define the coefficients

$$
a_{i j}^{n}(x, y)= \begin{cases}\delta_{i j} c_{n} \sigma_{n} w^{n}(x, y) & \text { if }(x, y) \in \Sigma^{n} \\ a_{i j} & \text { if }(x, y) \in \bar{\Omega}^{n},\end{cases}
$$

where $\delta_{i j}$ denotes the Kroneker symbol, $a_{i j}$ satisfy (2.6) and

$$
c_{n}>0, \quad \sigma_{n}=\frac{\alpha^{n}}{4^{n}} .
$$

We consider the bilinear form associated with the reinforcement problem

$$
a_{n}(u, v):=\int_{\widehat{\Omega}^{n}} \sum_{i, j=1}^{2} a_{i j}^{n} \frac{\partial u}{\partial x_{i}} \frac{\partial v}{\partial x_{j}} d x d y+\delta_{n} \int_{\widehat{\Omega}^{n}} u v d x d y
$$

where the coefficients $a_{i j}^{n}$ are defined in (3.3), (3.4), and $\delta_{n} \geq 0$. For every $n$, for $h=1,2$, we define $\left(\varphi_{h}\right)_{1, n}$ on $\overline{\Sigma_{1}^{n}}=\overline{\bigcup_{i \mid n} \Sigma_{1}^{i \mid n}}$

$$
\left(\varphi_{h}\right)_{1, n}(x, y)=G_{1}\left(\left(\varphi_{h}\right) \circ \psi_{i \mid n}\right) \circ \psi_{i \mid n}^{-1}(x, y) \quad \text { if }(x, y) \in \overline{\Sigma_{1}^{i \mid n}}
$$

where $G_{1}$ is the operator from $\operatorname{Lip}\left(K^{0}\right)$ to $\operatorname{Lip}\left(\bar{\Sigma}_{1}^{0}\right)$ defined in the following way. For every $\zeta \in(0,1)$, we define $P_{+}=P_{+}(\zeta)=\left(\zeta, \widehat{\eta}_{+}(\zeta)\right) \in \partial \Sigma_{1}^{0}$ to be the intersection of $\partial \Sigma_{1}^{0} \backslash K^{0}$ with the vertical line through the point $(\zeta, 0) \in K^{0}$. Then, for a given $g \in \operatorname{Lip}\left(K^{0}\right)$ we put

$$
G_{1}(g)(\zeta, \eta)= \begin{cases}g(0,0) & \text { if }(\zeta, \eta)=(0,0) \\ g(\zeta, 0) \frac{\widehat{\eta}_{+}-\eta}{\widehat{\eta}_{+}} & \text {if }(\zeta, \eta) \in \overline{\Sigma_{1}^{0}} \backslash\{A, B\} \\ g(1,0) & \text { if }(\zeta, \eta)=(1,0) .\end{cases}
$$

We construct, in a similar way, $G_{j}$ on $\overline{\Sigma_{j}^{0}}$ and $\left(\varphi_{h}\right)_{j, n}$ on $\overline{\Sigma_{j}^{n}}$ for $j=2,3$ with $h=1,2$. For every $n$, for $h=1,2$, we define

$$
\left(\varphi_{h}\right)_{n}(x, y)= \begin{cases}\varphi_{h}(x, y) & \text { if }(x, y) \in \Omega^{n} \\ \left(\varphi_{h}\right)_{j, n}(x, y) & \text { if }(x, y) \in \overline{\Sigma_{j}^{n}}\end{cases}
$$

Let

$$
\mathcal{K}_{n}=\left\{u \in H_{0}^{1}\left(\widehat{\Omega}^{n} ; w^{n}\right), u \geq\left(\varphi_{1}\right)_{n}\right\}
$$

and

$$
\mathcal{K}_{n}^{*}=\left\{u \in H_{0}^{1}\left(\widehat{\Omega}^{n} ; w^{n}\right), \quad\left(\varphi_{1}\right)_{n} \leq u \leq\left(\varphi_{2}\right)_{n}\right\} .
$$

Theorem 3.1 Let $c_{n}$ and $\sigma_{n}$ be as in (3.4). Then, for any $f_{n} \in L^{2}\left(\widehat{\Omega}^{n}\right), d_{n} \in \mathbb{R}$ there exists one and only one solution $u_{n}$ to the following problem

$$
\left\{\begin{array}{l}
\text { find } u_{n} \in \mathcal{K}_{n} \text { such that } \\
a_{n}\left(u_{n}, v-u_{n}\right) \geq \int_{\widehat{\Omega}^{n}} f_{n}\left(v-u_{n}\right) d x d y+\sigma_{n} d_{n} \int_{\partial \Omega^{n}}\left(v-u_{n}\right) d s \quad \forall v \in \mathcal{K}_{n},
\end{array}\right.
$$

where $a_{n}(\cdot, \cdot)$ is defined in (3.5). Moreover, $u_{n}$ is the only function that realizes the minimum of the energy functional

$$
\min _{v \in \mathcal{K}_{n}}\left\{a_{n}(v, v)-2 \int_{\widehat{\Omega}^{n}} f_{n} v d x d y-2 \sigma_{n} d_{n} \int_{\partial \Omega^{n}} v d s\right\} .
$$


Before proving Theorem 3.1, we recall a Poincaré type inequality where the relevant fact is that the constant $C_{P}$ is independent of $n$. We skip the proof because it is similar to the proof of Theorem 7.4 (following) (see also Theorem 6.1 in [13]).

Theorem 3.2 For any function $u \in H_{0}^{1}\left(\widehat{\Omega}^{n} ; w^{n}\right)$, the following estimate holds

$$
\|u\|_{L^{2}\left(\Sigma^{n}\right)}^{2} \leq \alpha^{-n} \int_{\Sigma^{n}}|\nabla u|^{2} w^{n} d x d y .
$$

Moreover, there exists a constant $C_{P}$ independent of $n$, such that,

$$
\|u\|_{L^{2}\left(\widehat{\Omega}^{n}\right)} \leq C_{P}\left(\|\nabla u\|_{L^{2}\left(\Omega^{n}\right)}^{2}+\sigma_{n} \int_{\Sigma^{n}}|\nabla u|^{2} w^{n} d x d y\right)^{1 / 2}
$$

for all $u \in H_{0}^{1}\left(\widehat{\Omega}^{n} ; w^{n}\right)$.

Proof of Theorem 3.1 As $\left(\varphi_{1}\right)_{n} \in \mathcal{K}_{n}$, the convex $\mathcal{K}_{n}$ is not empty. By Theorems 5.3 and 5.8 in [9], we have

$$
\left|\sigma_{n} d_{n} \int_{\partial \Omega^{n}} v d s\right| \leq C\left|d_{n}\right|\|v\|_{H^{1}\left(\Omega^{n}\right)}
$$

and

$$
\left|\int_{\widehat{\Omega}^{n}} f_{n} v d x d y\right| \leq\left\|f_{n}\right\|_{L^{2}\left(\widehat{\Omega}^{n}\right)}|| v \|_{L^{2}\left(\widehat{\Omega}^{n}\right)} .
$$

Moreover,

$$
\left|a_{n}(u, v)\right| \leq \max \left(\delta_{n}, c_{n} \sigma_{n}, \Lambda\right)\left\|\left.u\right|_{H_{0}^{1}\left(\widehat{\Omega}^{n} ; w^{n}\right)}|| v\right\|_{H_{0}^{1}\left(\widehat{\Omega}^{n} ; w^{n}\right)}
$$

and

$$
\|v\|_{H_{0}^{1}\left(\widehat{\Omega}^{n} ; w^{n}\right)}^{2} \leq\left(1+C_{P}^{2}\right) \max \left(\frac{1}{\lambda}, \frac{1}{c_{n} \sigma_{n}}\right) a_{n}(v, v) .
$$

Remark 3.1 Similar results hold for the two obstacle problems by considering now the convex $\mathcal{K}_{n}^{*}$. Moreover, the following estimate holds

$$
\left\|u_{n}\right\|_{L^{\infty}\left(\widehat{\Omega}^{n}\right)} \leq \max \left(\left\|\varphi_{1}\right\|_{C^{0}(\bar{\Omega})},\left\|\varphi_{2}\right\|_{C^{0}(\bar{\Omega})} .\right.
$$

\section{Mosco convergence}

We consider the sequence of weighted energy functionals in $L^{2}\left(\widehat{\Omega}^{1}\right)$

$$
F^{n}[u]= \begin{cases}\int_{\widehat{\Omega}^{n}} \sum_{i, j=1}^{2} a_{i j}^{n} \frac{\partial u}{\partial x_{i}} \frac{\partial u}{\partial x_{j}} d x d y+\delta_{n} \int_{\widehat{\Omega}^{n}} u^{2} d x d y & \text { if }\left.u\right|_{\widehat{\Omega}^{n}} \in H_{0}^{1}\left(\widehat{\Omega}^{n} ; w^{n}\right) \\ +\infty & \text { otherwise in } L^{2}\left(\widehat{\Omega}^{1}\right)\end{cases}
$$

[the coefficients $a_{i j}^{n}$ are defined in (2.6), (3.3), (3.4), $\delta_{n} \geq 0$ ] and

$$
\begin{aligned}
& F_{c_{0}}[u] \\
& \quad= \begin{cases}\int_{\Omega} \sum_{i, j=1}^{2} a_{i j} \frac{\partial u}{\partial x_{i}} \frac{\partial u}{\partial x_{j}} d x d y+\delta_{0} \int_{\Omega} u^{2} d x d y+c_{0} \int_{\partial \Omega} u^{2} d \mu & \text { if }\left.u\right|_{\Omega} \in H^{1}(\Omega) \\
+\infty & \text { otherwise in } L^{2}\left(\widehat{\Omega}^{1}\right) .\end{cases}
\end{aligned}
$$


We recall the notion of $M$-convergence of functionals, introduced in [24] (see also [25]).

Definition 4.1 A sequence of functionals $F^{n}: H \rightarrow(-\infty,+\infty$ ] is said to $M$-converge to a functional $F: H \rightarrow(-\infty,+\infty]$ in a Hilbert space $H$, if

(a) For every $u \in H$ there exists $u_{n}$ converging strongly to $u$ in $H$ such that

$$
\lim \sup F^{n}\left[u_{n}\right] \leq F[u], \text { as } n \rightarrow+\infty \text {. }
$$

(b) For every $v_{n}$ converging weakly to $u$ in $H$

$$
\liminf F^{n}\left[v_{n}\right] \geq F[u], \quad \text { as } n \rightarrow+\infty \text {. }
$$

In order to study the asymptotic behaviour, we fix the further assumptions

$$
\begin{aligned}
& \delta_{n} \geq 0 \text { and } \delta_{n} \rightarrow \delta_{0} \text { as } n \rightarrow+\infty, \\
& c_{n}>0 \text { and } c_{n} \rightarrow c_{0} \text { as } n \rightarrow+\infty .
\end{aligned}
$$

Theorem 4.1 Let us assume (4.5) and (4.6). Then, the sequence of functionals $F^{n}$, defined in (4.1), $M$-converges in $L^{2}\left(\widehat{\Omega}^{1}\right)$ to the functional $F_{c_{0}}$ defined in (4.2) as $n \rightarrow+\infty$.

Before proving Theorem 4.1 we recall the following convergence result that we shall use several times from now on (see Proposition 4.1 in [13]).

Proposition 4.1 Let $\sigma_{n}$ be as in (3.4). Then, for every sequence $g_{n} \in H^{1}(\Omega)$ weakly converging to $g^{*}$ in $H^{1}(\Omega)$, we have

$$
\sigma_{n} \int_{\partial \Omega^{n}} g_{n} d s \rightarrow \int_{\partial \Omega} g^{*} d \mu, \text { as } n \rightarrow+\infty .
$$

Proof of Theorem 4.1 This theorem can be proved just as Theorem 4.1 was proved in [13]. The coefficients of the forms are different, as is the geometry of the layers: however, since these peculiarities do not change the basic proof, here we only highlight some crucial points and we refer the reader to the proof of Theorem 4.1 in [13] for details. First, we proceed with the proof of condition (a) in Definition 4.1. We consider a given function $u$ as in condition (a) and we observe that, without loss of generality, we can assume that $\left.u\right|_{\Omega} \in H^{1}(\Omega)$, otherwise the inequality (4.3) becomes trivial. We assume, in addition, that $\left.u\right|_{\bar{\Omega}} \in \operatorname{Lip}(\bar{\Omega})$. We construct, as in Sect. 3, $G_{j}$ on $\overline{\Sigma_{j}^{0}}$ and $u_{j, n}$ on $\overline{\Sigma_{j}^{n}}$ for $j=1,2,3$ [see (3.6) and (3.7)]. For every $n$, we define

$$
u_{n}(x, y)= \begin{cases}u(x, y) & \text { if }(x, y) \in \Omega^{n} \\ u_{j, n}(x, y) & \text { if }(x, y) \in \overline{\Sigma_{j}^{n}}\end{cases}
$$

We denote by $\bar{u}$ the extension to zero of $u$ outside $\Omega$ and by $\bar{u}_{n}$ the extension to zero of $u_{n}$ outside $\widehat{\Omega}^{n}$. We note that $\bar{u}_{n}$ tend to $\bar{u}$ in $L^{2}\left(\widehat{\Omega}^{1}\right)$, $\sup _{\bar{\Sigma}^{n}}\left|\bar{u}_{n}\right| \leqslant \sup _{\bar{\Omega}}|u|$ and the functions $u_{n}$ defined in (4.8) belong to $H_{0}^{1}\left(\widehat{\Omega}^{n}, w_{n}\right)$.

For each $n$, we split the integral $F^{n}\left[u_{n}\right]$ in three terms, taking into account the definitions of $a_{i j}^{n}$ and $u_{n}$,

$$
F^{n}\left[u_{n}\right]=\int_{\Omega^{n}} \sum_{i, j=1}^{2} a_{i j} \frac{\partial u}{\partial x_{i}} \frac{\partial u}{\partial x_{j}} d x d y+\delta_{n} \int_{\widehat{\Omega}^{n}} u_{n}^{2} d x d y+\sigma_{n} c_{n} \int_{\Sigma^{n}}\left|\nabla u_{n}\right|^{2} w^{n} d x d y
$$


Since the sets $\Omega^{n}$ tend to the set $\Omega$ as $n \rightarrow+\infty$, we get

$$
\begin{aligned}
& \lim _{n \rightarrow+\infty} \int_{\Omega^{n}} \sum_{i, j=1}^{2} a_{i j} \frac{\partial u}{\partial x_{i}} \frac{\partial u}{\partial x_{j}} d x d y=\int_{\Omega} \sum_{i, j=1}^{2} a_{i j} \frac{\partial u}{\partial x_{i}} \frac{\partial u}{\partial x_{j}} d x d y, \\
& \lim _{n \rightarrow+\infty} \delta_{n} \int_{\Omega^{n}} u^{2} d x d y=\delta_{0} \int_{\Omega} u^{2} d x d y, \\
& \lim _{n \rightarrow+\infty} \delta_{n} \int_{\Sigma^{n}} u_{n}^{2} d x d y=0 .
\end{aligned}
$$

Finally, as in Theorem 4.1 in [13] we can show

$$
\lim _{n \rightarrow+\infty} c_{n} \sigma_{n} \int_{\Sigma^{n}}\left|\nabla u_{n}\right|^{2} w^{n} d x d y=c_{0} \int_{\partial \Omega} u^{2} d \mu .
$$

We complete the proof of part (a) of the Theorem by making use of the diagonal formula of Corollary 1.16 of [2].

Now we prove condition (b) of Definition 4.1. Let $v_{n}$ be a sequence as in (b), that is,

$$
v_{n} \rightarrow u \text { in } L^{2}\left(\widehat{\Omega}^{1}\right) .
$$

In order to prove the inequality (4.4), it is not restrictive to assume that

$$
\liminf F^{n}\left[v_{n}\right] \leq C^{*}<+\infty .
$$

Then, from (4.13) and (4.14), up to passing to a subsequence, we deduce that

$$
\left\|v_{n}\right\|_{H^{1}\left(\Omega^{n}\right)} \leq C
$$

where $C$ is a constant independent of $n$. By Theorem 5.7 in [9], there exists a bounded linear extension operator Ext $t_{J}: H^{1}\left(\Omega^{n}\right) \rightarrow H^{1}\left(\mathbb{R}^{2}\right)$, whose norm is independent of $n$, that is,

$$
\left\|E x t_{J} v_{n}\right\|_{H^{1}\left(\mathbb{R}^{2}\right)} \leqslant C_{J}\left\|v_{n}\right\|_{H^{1}\left(\Omega^{n}\right)}
$$

with $C_{J}$ independent of $n$. We put

$$
\hat{v}_{n}=\left.\left(\left.\operatorname{Ext}_{J} v_{n}\right|_{\Omega^{n}}\right)\right|_{\Omega},
$$

then there exists $\hat{v} \in H^{1}(\Omega)$ and a subsequence of $\hat{v}_{n}$, denoted by $\hat{v}_{n}$ again, weakly converging to $\hat{v}$ in $H^{1}(\Omega)$. By a direct calculation, we can prove that the sequence $\hat{v}_{n}$ weakly converges to $u$ in $L^{2}(\Omega)$ hence

$$
\begin{aligned}
& \left.\hat{v}_{n} \rightarrow u\right|_{\Omega} \text { in } H^{1}(\Omega), \\
& \liminf _{n \rightarrow+\infty} \int_{\Omega^{n}}\left|\nabla v_{n}\right|^{2} d x d y \geq \int_{\Omega}|\nabla u|^{2} d x d y
\end{aligned}
$$

and

$$
\lim _{n \rightarrow+\infty} \delta_{n} \int_{\Omega^{n}} v_{n}^{2} d x d y=\delta_{0} \int_{\Omega} u^{2} d x d y
$$

Finally, as in Theorem 4.1 in [13] we show that (if $c_{0}>0$ )

$$
\liminf _{n \rightarrow+\infty} c_{n} \sigma_{n} \int_{\Sigma^{n}}\left|\nabla v_{n}\right|^{2} w^{n} d x d y \geq c_{0} \int_{\partial \Omega} u^{2} d \mu
$$


Remark 4.1 We note if $u \in \operatorname{Lip}(\bar{\Omega}) \cap \mathcal{K}\left(u \in \operatorname{Lip}(\bar{\Omega}) \cap \mathcal{K}^{*}\right)$, then the function $u_{n}$ defined in (4.8) belongs to $\mathcal{K}_{n}\left(\mathcal{K}_{n}^{*}\right)$. Then, by making use of the diagonal formula of Corollary 1.16 of [2], we can deduce that for any $u \in \mathcal{K}\left(u \in \mathcal{K}^{*}\right)$ the corresponding $u_{n}$ belongs to $\mathcal{K}_{n}\left(\mathcal{K}_{n}^{*}\right)$.

When the conductivity of the thin fibers vanishes more slowly than the thickness of the fiber, that is,

$$
c_{n} w^{n} \rightarrow 0, \quad c_{n} \rightarrow+\infty
$$

we introduce the limit functional (4.22) in $L^{2}\left(\widehat{\Omega}^{1}\right)$

$$
F_{\infty}[u]=\left\{\begin{array}{ll}
\int_{\Omega}|\nabla u|^{2} d x d y+\delta_{0} \int_{\Omega} u^{2} d x d y & \text { if }\left.u\right|_{\Omega} \in H_{0}^{1}(\Omega) \\
+\infty & \text { otherwise in } L^{2}\left(\widehat{\Omega}^{1}\right)
\end{array} .\right.
$$

The following theorem can be proved just as Theorem 4.2 was proved in [13].

Theorem 4.2 Let us assume (4.21) and (4.5). Then the sequence of functionals $F^{n}$, defined in (4.1), $M$-converges in $L^{2}\left(\widehat{\Omega}^{1}\right)$ as $n \rightarrow+\infty$ to the energy functional $F_{\infty}[u]$ defined in (4.22).

\section{Asymptotics}

In order to study the asymptotic behaviour of the functions $u_{n}$, we assume that

$$
\begin{aligned}
& f_{n}, f \in L^{2}\left(\widehat{\Omega}^{1}\right), \text { and } f_{n} \rightarrow f \in L^{2}\left(\widehat{\Omega}^{1}\right), \quad \text { as } n \rightarrow+\infty ; \\
& d_{n}, d \in \mathbb{R}, \text { and } d_{n} \rightarrow d \text { as } n \rightarrow+\infty .
\end{aligned}
$$

Our first result concerns the case of thin fibers whose conductivity vanishes more slowly than the thickness of the fiber. The cases in which the conductivity of the thin fibers vanishes at the same rate as the thickness of the fiber or more quickly will be taken into account in Theorems 5.2 (coercive case), and 5.3 (semicoercive).

\subsection{Coercive case}

When the conductivity of the thin fibers vanishes more slowly than the thickness of the fiber, we state the following Theorem 5.1 (see also Theorem 3.3 in [13]).

Theorem 5.1 Let us assume (4.5), (4.21), (5.1), and (5.2). Then the sequence of the solutions $u_{n}\left[\right.$ defined in (3.11)] converges to the function $u$ [defined in (2.8)] weakly in $H_{l o c}^{1}(\Omega)$ and strongly in $L^{2}(\Omega)$.

The following theorem deals with thin fibers whose conductivity vanishes at the same rate as the thickness of the fiber or more quickly.

Theorem 5.2 Let us assume conditions (2.14), (4.5), (4.6), (5.1), and (5.2). Then the sequence of the solutions $u_{n}$ [defined in (3.11)] converges to the function $u$ [defined in (2.16)] weakly in $H_{l o c}^{1}(\Omega)$, and weakly in $L^{2}(\Omega)$. Moreover, if

$$
\lim _{n \rightarrow+\infty} \sigma_{n} c_{n} \alpha^{n}=+\infty
$$

then the sequence of the solutions $u_{n}$ converges to the function $u$ strongly in $L^{2}(\Omega)$. 
Proof Let $u_{n}$ be the solution to the problem (3.11). Then

$$
a_{n}\left(u_{n}, u_{n}\right) \leq\left\|f_{n}\right\|_{L^{2}\left(\widehat{\Omega}^{n}\right)}\left\|u_{n}-v_{n}\right\|_{L^{2}\left(\widehat{\Omega}^{n}\right)}+C\left|d_{n}\right|\left\|u_{n}-v_{n}\right\|_{H^{1}\left(\Omega^{n}\right)}+a_{n}\left(u_{n}, v_{n}\right)
$$

where we have used Theorems 5.3 and 5.7 in [9]. Suppose first that $\delta_{0}>0$. Then,

$$
\begin{aligned}
& \int_{\Omega^{n}}\left|\nabla u_{n}\right|^{2} d x d y+c_{n} \sigma_{n} \int_{\Sigma^{n}}\left|\nabla u_{n}\right|^{2} w_{n} d x d y+\int_{\widehat{\Omega}^{n}}\left|u_{n}\right|^{2} d x d y \\
& \quad \leq \frac{1}{\lambda} \int_{\Omega^{n}} a_{i j} \frac{\partial u}{\partial x_{i}} \frac{\partial u}{\partial x_{j}} d x d y+c_{n} \sigma_{n} \int_{\Sigma^{n}}\left|\nabla u_{n}\right|^{2} w_{n} d x d y+\frac{2 \delta_{n}}{\delta_{0}} \int_{\widehat{\Omega}^{n}}\left|u_{n}\right|^{2} d x d y \\
& \quad \leq \max \left(\frac{1}{\lambda}, \frac{2}{\delta_{0}}, 1\right) a_{n}\left(u_{n}, u_{n}\right) .
\end{aligned}
$$

The right end side of inequality (5.4) can be estimated as follows

$$
\begin{aligned}
& \left\|f_{n}\right\|_{L^{2}\left(\widehat{\Omega}^{n}\right)}|| u_{n}-v_{n}\left\|_{L^{2}\left(\widehat{\Omega}^{n}\right)}+C\left|d_{n}\right||| u_{n}-v_{n}\right\|_{H^{1}\left(\Omega^{n}\right)}+a_{n}\left(u_{n}, v_{n}\right) \\
& \leq\left\|f_{n}\right\|_{L^{2}\left(\widehat{\Omega}^{n}\right)}\left(\left\|u_{n}\right\|+\left\|v_{n}\right\|_{L^{2}\left(\widehat{\Omega}^{n}\right)}\right)+C\left|d_{n}\right|\left(|| u_{n}\|+\| v_{n} \|_{H^{1}\left(\Omega^{n}\right)}\right) \\
& \quad+\Lambda\left\|\nabla u_{n}\right\|_{L^{2}\left(\Omega^{n}\right)}|| \nabla v_{n} \|_{L^{2}\left(\Omega^{n}\right)}+c_{n} \sigma_{n}\left(\int_{\Sigma^{n}}\left|\nabla u_{n}\right|^{2} w_{n} d x d y\right)^{\frac{1}{2}} \\
& \quad \times\left(\int_{\Sigma^{n}}\left|\nabla v_{n}\right|^{2} w_{n} d x d y\right)^{\frac{1}{2}} .
\end{aligned}
$$

We choose as test function $v_{n}=\left(\varphi_{1}\right)_{n}$ and we obtain

$$
\begin{aligned}
& \left\|v_{n}\right\|_{L^{2}\left(\widehat{\Omega}^{n}\right)}^{2} \leq\left\|\varphi_{1}\right\|_{L^{2}(\Omega)}^{2}+\sup _{\bar{\Omega}}\left|\varphi_{1}\right|^{2}\left|\Sigma_{n}\right|, \\
& \left\|\nabla v_{n}\right\|_{L^{2}\left(\Omega^{n}\right)}^{2} \leq\left\|\nabla \varphi_{1}\right\|_{L^{2}(\Omega)}^{2} .
\end{aligned}
$$

Moreover, by (4.12), we deduce

$$
c_{n} \sigma_{n} \int_{\Sigma^{n}}\left|\nabla v_{n}\right|^{2} w^{n} d x d y \leq \max \left(1,2 c_{0} \int_{\partial \Omega}\left|\varphi_{1}\right|^{2} d \mu\right) .
$$

By using the previous inequalities (5.4)-(5.9) we obtain

$$
\begin{aligned}
& \int_{\Omega^{n}}\left|\nabla u_{n}\right|^{2} d x d y+c_{n} \sigma_{n} \int_{\Sigma^{n}}\left|\nabla u_{n}\right|^{2} w_{n} d x d y \\
& \quad \leqslant C_{1}\left(\delta_{0}, \lambda, \Lambda,|d|,|| f\left\|_{L^{2}\left(\widehat{\Omega}^{1}\right)},\right\| \varphi_{1} \|_{C^{1}(\bar{\Omega})}\right)
\end{aligned}
$$

and

$$
\left\|u_{n}\right\|_{L^{2}\left(\widehat{\Omega}^{n}\right)}^{2} \leqslant C_{1}\left(\delta_{0}, \lambda, \Lambda,|d|,\|f\|_{L^{2}\left(\widehat{\Omega}^{1}\right)},\left\|\varphi_{1}\right\|_{C^{1}(\bar{\Omega})}\right)
$$

where the constant $C_{1}$ does not depend on $n$.

By assumption (2.14), if $\delta_{0}=0$, then $c_{0}>0$ : in this case, by using Theorem 3.2, we have 


$$
\begin{aligned}
& \int_{\Omega^{n}}\left|\nabla u_{n}\right|^{2} d x d y+c_{n} \sigma_{n} \int_{\Sigma^{n}}\left|\nabla u_{n}\right|^{2} w_{n} d x d y+\int_{\widehat{\Omega}^{n}}\left|u_{n}\right|^{2} d x d y \\
& \quad \leq \frac{1+C_{p}^{2}}{\lambda} \int_{\Omega^{n}} a_{i j} \frac{\partial u_{n}}{\partial x_{i}} \frac{\partial u_{n}}{\partial x_{j}} d x d y+c_{n} \sigma_{n} \frac{2 C_{p}^{2}+c_{0}}{c_{0}} \int_{\Sigma^{n}}\left|\nabla u_{n}\right|^{2} w_{n} d x d y \\
& \quad \leq \max \left(\frac{1+C_{p}^{2}}{\lambda}, \frac{2 C_{p}^{2}+c_{0}}{c_{0}}\right) a_{n}\left(u_{n}, u_{n}\right) .
\end{aligned}
$$

Proceeding as before,

$$
\int_{\Omega^{n}}\left|\nabla u_{n}\right|^{2} d x d y+c_{n} \sigma_{n} \int_{\Sigma^{n}}\left|\nabla u_{n}\right|^{2} w_{n} d x d y \leq C_{2}\left(c_{0}, \lambda, \Lambda,|d|,\|f\|_{L^{2}\left(\widehat{\Omega}^{1}\right)},\left\|\varphi_{1}\right\|_{C^{1}(\bar{\Omega})}\right),
$$

and

$$
\left\|u_{n}\right\|_{L^{2}\left(\widehat{\Omega}^{n}\right)}^{2} \leqslant C_{2}\left(c_{0}, \lambda, \Lambda,|d|,\|f\|_{L^{2}\left(\widehat{\Omega}^{1}\right)},\left\|\varphi_{1}\right\|_{C^{1}(\bar{\Omega})}\right)
$$

where the constant $C_{2}$ does not depend on $n$. We consider the function $u_{n}^{*}$, which is a suitable extension of the function $u_{n}$ from the set $\Omega^{n}$ to the set $\widehat{\Omega}^{1}$ (we use an extension operator whose norm is independent of the increasing number of sides, see Theorem 5.7 in [9]) and for every $n$, from either (5.10) and (5.11) or (5.13) and (5.14), we derive

$$
\left\|u_{n}^{*}\right\|_{H^{1}\left(\widehat{\Omega}^{1}\right)} \leq C_{J}\left\|u_{n}\right\|_{H^{1}\left(\Omega^{n}\right)} \leq C .
$$

Therefore, there exists a subsequence still denoted by $u_{n}^{*}$ that weakly converges to a function $u^{*}$ in $H^{1}\left(\widehat{\Omega}^{1}\right)$. Now we prove that $\left.u^{*}\right|_{\Omega}=u$. By using condition $(b)$ of M-convergence and Proposition 4.1, we obtain that

$$
\begin{aligned}
& F_{c_{0}}\left[u^{*}\right]-2 \int_{\Omega} f u^{*} d x d y-2 d \int_{\partial \Omega} u^{*} d \mu \\
& \quad \leq \liminf \left(F^{n}\left[u_{n}^{*}\right]-2 \int_{\widehat{\Omega}^{n}} f_{n} u_{n}^{*} d x d y-2 \sigma_{n} d_{n} \int_{\partial \Omega^{n}} u_{n}^{*} d s\right) .
\end{aligned}
$$

By using condition (a) of M-convergence there exists $v_{n} \in L^{2}\left(\widehat{\Omega}^{1}\right)$ [defined as in (4.8) in the proof of Theorem 4.1] converging strongly in $L^{2}\left(\widehat{\Omega}^{1}\right)$ to $\bar{u}$ such that

$$
\lim F^{n}\left[v_{n}\right]=F_{c_{0}}[\bar{u}]=F_{c_{0}}[u],
$$

as $n \rightarrow+\infty$. We recall that we denote by $\bar{u}$ the extension to zero of $u$ outside $\Omega$. Moreover, by Remark $\left.4.1 v_{n}\right|_{\widehat{\Omega}^{n}} \in \mathcal{K}_{n}$. Then by Proposition 4.1 (where $g_{n}$ is a suitable extension of $v_{n}$ ) we obtain

$$
\begin{aligned}
& \lim \left(F^{n}\left[v_{n}\right]-2 \sigma_{n} d_{n} \int_{\partial \Omega^{n}} v_{n} d s-2 \int_{\widehat{\Omega}^{n}} f_{n} v_{n} d x d y\right) \\
& =F_{c_{0}}[u]-2 d \int_{\partial \Omega} u d \mu-2 \int_{\Omega} f u d x d y .
\end{aligned}
$$


Then

$$
\begin{aligned}
F^{n} & {\left[u_{n}\right]-2 \sigma_{n} d_{n} \int_{\partial \Omega^{n}} u_{n} d s-2 \int_{\widehat{\Omega}^{n}} f_{n} u_{n} d x d y } \\
& =\min _{v \in \mathcal{K}_{n}}\left(F^{n}[v]-2 \sigma_{n} d_{n} \int_{\partial \Omega^{n}} v d s-2 \int_{\widehat{\Omega}^{n}} f_{n} v d x d y\right) \\
& \leq F^{n}\left[v_{n}\right]-2 \sigma_{n} d_{n} \int_{\partial \Omega^{n}} v_{n} d s-2 \int_{\widehat{\Omega}^{n}} f_{n} v_{n} d x d y .
\end{aligned}
$$

Just as we obtain, by direct calculations,

$$
\begin{aligned}
& \liminf \left(F^{n}\left[u_{n}^{*}\right]-2 \sigma_{n} d_{n} \int_{\partial \Omega^{n}} u_{n}^{*} d s-2 \int_{\widehat{\Omega}^{n}} f_{n} u_{n}^{*} d x d y\right) \\
& \quad \leq \liminf \left(F^{n}\left[u_{n}\right]-2 \sigma_{n} d_{n} \int_{\partial \Omega^{n}} u_{n} d s-2 \int_{\widehat{\Omega}^{n}} f_{n} u_{n} d x d y\right)
\end{aligned}
$$

by combining (5.16)-(5.19) we obtain that

$$
F_{c_{0}}\left[u^{*}\right]-2 \int_{\Omega} f u^{*} d x d y-2 d \int_{\partial \Omega} u^{*} d \mu \leq F_{c_{0}}[u]-2 \int_{\Omega} f u d x d y-2 d \int_{\partial \Omega} u d \mu .
$$

By the uniqueness of the solution (2.16), we conclude that $\left.u^{*}\right|_{\Omega}=u$, and $\left.u_{n}^{*}\right|_{\Omega}$ converges to $u$ weakly in $H^{1}(\Omega)$. As the $u_{n}^{*}=u_{n}$ in $\Omega_{N}$ with $n \geq N$ then $u_{n}$ converges to $u$ weakly in $H_{l o c}^{1}(\Omega)$ and from (5.11) or (5.14) we deduce that $u_{n}$ converge to $u$ weakly in $L^{2}(\Omega)$. Moreover, if we assume condition (5.3), then the strong convergence holds in $L^{2}(\Omega)$ : in fact,

$$
\begin{gathered}
\lim \sup \int_{\Omega} u_{n}^{2} d x d y \leq \lim \sup \left(\int_{\Omega^{n}} u_{n}^{2} d x d y+\int_{\Sigma^{n}} u_{n}^{2} d x d y\right) \\
\leq \lim \sup \left(\int_{\Omega}\left(u_{n}^{*}\right)^{2} d x d y+\int_{\Sigma^{n}} u_{n}^{2} d x d y\right)=\int_{\Omega} u^{2} d x d y
\end{gathered}
$$

where we have used the strong convergence of the sequence $u_{n}^{*}$ to $u$ in the space $L^{2}(\Omega)$ and estimates (3.13), (5.3), and (5.10) or (5.13).

Remark 5.1 The results of Theorems 5.1 and 5.2 apply also to the case of equations by the same proof and they improve the results of Theorems 3.1 and 3.3 in [13]. In fact, in the present paper, the assumptions are weaker than the assumptions of [13]. Moreover, we prove the convergence in $L^{2}(\Omega)$ directly for the solutions rather than for suitable extensions of the solutions.

Remark 5.2 The results of Theorems 5.1 and 5.2 hold for the two obstacle problems where $\mathcal{K}^{*}$ and $\mathcal{K}_{n}^{*}$ are defined respectively in (2.20) and (3.10). Moreover, in this case, from estimates (3.16) and (5.15) we obtain strong convergence in $L^{p}(\Omega)$ with $p<+\infty$.

In the next subsection, we establish results without requiring condition (2.14).

\subsection{Semicoercive case}

We note that, in the assumptions and notation of Sect. 2, conditions (2.23) and (2.24) (as well as analogous conditions in Remarks $2.3,2.4$ ) guarantee the existence and uniqueness of the solution of obstacle problems (see Theorem 2.3). In this subsection, we discuss assumptions on the approximation data $f_{n}$ and on the coefficients $c_{n}$ that guarantee asymptotic results. In particular, we can extend $f$ to zero outside $\Omega_{n}$ or, according to the classical setting (see 
Theorem 9.3 in [3]), we can impose some conditions on the vanishing rate of the sequence $c_{n}$ (for a complete discussion, see Remarks 5.3 and 5.4 following).

Theorem 5.3 Let us assume conditions (4.6) with $c_{0}=0$, (4.5) with $\delta_{0}=0$, (2.23), (2.24), (5.1), (5.2), and

$$
\frac{\left\|f_{n}\right\|_{L^{2}\left(\Sigma^{n}\right)}^{2}}{c_{n} \sigma_{n} \alpha^{n}} \leq c^{*}
$$

with $c^{*}>0$.

Then the sequence of the solutions $u_{n}$ [defined in (3.11)] converges to the function $u$ [defined in (2.25)] weakly in $H_{l o c}^{1}(\Omega)$. If

$$
c_{n} \sigma_{n} \alpha^{n} \geq c^{* *}
$$

for some $c^{* *}>0$ and thus

$$
\frac{\left\|f_{n}\right\|_{L^{2}\left(\Sigma^{n}\right)}^{2}}{c_{n} \sigma_{n} \alpha^{n}} \rightarrow 0,
$$

then the solutions $u_{n}$ [defined in (3.11)] converge weakly in $L^{2}(\Omega)$. Moreover, if assumption (5.3) holds, then the solutions $u_{n}$ converge strongly in $L^{2}(\Omega)$.

Proof As $\delta_{n}$ tends to zero, we assume, for the sake of simplicity, that $\delta_{n}=0$. From (2.23) we deduce, by taking into account (3.8) and (3.9), that $0 \in \mathcal{K}_{n}$ and

$$
a_{n}\left(u_{n}, u_{n}\right) \leq \int_{\widehat{\Omega}^{n}} f_{n} u_{n} d x d y+d_{n} \sigma_{n} \int_{\partial \Omega^{n}} u_{n} d s .
$$

Bearing in mind the proof of Theorem 5.2, we show that there exists a constant $C$ such that

$$
\left\|u_{n}\right\|_{H^{1}\left(\Omega^{n}\right)} \leq C
$$

where the functions $u_{n}$ are the solutions defined in (3.11). Suppose the statement to be proved is false: for every $m$ in $\mathbb{N}$, there exists $u_{n_{m}}$ that we shall denote, from now on, simply by $u_{m}$ such that

$$
A_{m}^{2}:=\left\|u_{m}\right\|_{H^{1}\left(\Omega^{m}\right)}^{2} \geq m^{2}
$$

and

$$
a_{m}\left(u_{m}, u_{m}\right) \leq \int_{\widehat{\Omega}^{m}} f_{m} u_{m} d x d y+d_{m} \sigma_{m} \int_{\partial \Omega^{m}} u_{m} d s
$$

Set

$$
v_{m}=\frac{u_{m}}{A_{m}}:
$$

we have

$$
\left\|v_{m}\right\|_{H^{1}\left(\Omega^{m}\right)}^{2}=1 .
$$

We denote by $v_{m}^{*}$ an extension of $v_{m_{\mid \Omega^{n}}}$ to $\widehat{\Omega}^{1}$ such that

$$
\left\|v_{m}^{*}\right\|_{H^{1}\left(\widehat{\Omega}^{1}\right)}^{2} \leq C
$$


with $C$ independent to $m$ (see Theorem 5.7 in [9]). Then, there exists a function $v^{*} \in H^{1}\left(\widehat{\Omega}^{1}\right)$ and a subsequence (still denoted by $v_{m}^{*}$ ) that converges to $v^{*}$ weakly in $H^{1}\left(\widehat{\Omega}^{1}\right)$ (and strongly in $H^{s}\left(\widehat{\Omega}^{1}\right)$ for $\left.0 \leq s<1\right)$. From (5.23) we deduce

$$
\begin{aligned}
& \lambda\left\|\nabla v_{m}\right\|_{L^{2}\left(\Omega^{m}\right)}^{2}+c_{m} \sigma_{m} \int_{\Sigma^{m}}\left|\nabla v_{m}\right|^{2} w^{m} d x d y \\
& \leq \frac{1}{A_{m}}\left(\int_{\widehat{\Omega}^{m}} f_{m} v_{m} d x d y+d_{m} \sigma_{m} \int_{\partial \Omega^{m}} v_{m} d s\right) .
\end{aligned}
$$

As

$$
\left|d_{m} \sigma_{m} \int_{\partial \Omega^{m}} v_{m} d s\right| \leq C|d|
$$

we have to estimate

$$
\int_{\widehat{\Omega}^{m}} f_{m} v_{m} d x d y
$$

We start by noting that

$$
\left|\int_{\Omega^{m}} f_{m} v_{m} d x d y\right| \leq C\|f\|_{L^{2}(\Omega)},
$$

hence, we have to estimate the part on $\Sigma^{m}$; by taking into account statement (3.13) we obtain

$$
\begin{aligned}
\left|\int_{\Sigma^{m}} f_{m} v_{m} d x d y\right| & \leq\left\|f_{m}\right\|_{L^{2}\left(\Sigma^{m}\right)}\left(\int_{\Sigma^{m}} v_{m}^{2} d x d y\right)^{1 / 2} \\
& \leq\left\|f_{m}\right\|_{L^{2}\left(\Sigma^{m}\right)}\left(\frac{c_{m} \sigma_{m}}{c_{m} \sigma_{m} \alpha^{m}} \int_{\Sigma^{m}}\left|\nabla v_{m}\right|^{2} w^{m} d x d y\right)^{1 / 2}
\end{aligned}
$$

and by (5.29)

$$
\begin{aligned}
& \lambda\left\|\nabla v_{m}\right\|_{L^{2}\left(\Omega^{m}\right)}^{2}+c_{m} \sigma_{m} \int_{\Sigma^{m}}\left|\nabla v_{m}\right|^{2} w^{m} d x d y \\
& \quad \leq \frac{1}{A_{m}}\left(\int_{\Omega^{m}} f_{m} v_{m} d x d y+C+\frac{\left\|f_{m}\right\|_{L^{2}\left(\Sigma^{m}\right)}^{2}}{c_{m} \sigma_{m} \alpha^{m}} c_{m} \sigma_{m} \int_{\Sigma^{m}}\left|\nabla v_{m}\right|^{2} w^{m} d x d y\right) .
\end{aligned}
$$

Hence, by (5.20) and (5.25),

$$
\lambda\left\|\nabla v_{m}\right\|_{L^{2}\left(\Omega^{m}\right)}^{2}+c_{m} \sigma_{m} \int_{\Sigma^{m}}\left|\nabla v_{m}\right|^{2} w^{m} d x d y \leq \frac{C}{A_{m}} .
$$

From (5.30), by the weak lower semicontinuity of the norm, we obtain, for any fixed $N_{0}$,

$$
\left\|\nabla v^{*}\right\|_{L^{2}\left(\Omega^{\left.N_{0}\right)}\right.}^{2} \leq \liminf _{m}\left\|\nabla v_{m}\right\|_{L^{2}\left(\Omega^{\left.N_{0}\right)}\right.}^{2}=0
$$

then $v^{*}=k_{1}$ a. e. in $\Omega^{N_{0}}$. We observe that $k_{1} \geq 0$ in fact by construction $v_{m} \geq \frac{1}{A_{m}} \min _{\bar{\Omega}}\left(\varphi_{1}\right)$. As $N_{0}$ is arbitrary, we deduce that $v^{*}=k_{1}$ a. e. in $\Omega$ and $\nabla v_{m}^{*}$ weakly converges to 0 in $L^{2}(\Omega)$. We show that $k_{1}=0$ : if $k_{1}>0$ we obtain a contradiction with (2.24). In fact, from (5.29) we obtain

$$
0 \leq \int_{\widehat{\Omega}^{m}} f_{m} v_{m} d x d y+d_{m} \sigma_{m} \int_{\partial \Omega^{m}} v_{m} d s
$$


and

$$
\int_{\widehat{\Omega}^{m}} f_{m} v_{m} d x d y=\int_{\Omega^{m}} f_{m} v_{m} d x d y+\int_{\Sigma^{m}} f_{m} v_{m} d x d y .
$$

We estimate $\int_{\Sigma^{m}} f_{m} v_{m} d x d y$. As previously, we obtain

$$
\left|\int_{\Sigma^{m}} f_{m} v_{m} d x d y\right| \leq|| f_{m} \|_{L^{2}\left(\Sigma^{m}\right)}\left(\frac{c_{m} \sigma_{m}}{c_{m} \sigma_{m} \alpha^{m}} \int_{\Sigma^{m}}\left|\nabla v_{m}\right|^{2} w^{m} d x d y\right)^{1 / 2}
$$

and hence, by (5.20) and (5.30),

$$
\int_{\Sigma^{m}} f_{m} v_{m} d x d y \rightarrow 0 .
$$

As the term $\int_{\Sigma^{m}} f_{m} v_{m}^{*} d x d y$ tends to 0 by the strong convergence of the functions $v_{m}^{*}$ in $L^{p}(\Omega), p>2$, and by (4.7), (5.1), and (5.2), from (5.32) we deduce

$$
0 \leq k_{1}\left(\int_{\Omega} f d x d y+d \int_{\partial \Omega} d \mu\right)
$$

that is a contradiction with (2.24). On the other hand, if $k_{1}=0$, we have a contradiction with (5.27). In fact, by (5.27)

$$
1=\left\|v_{m}\right\|_{L^{2}\left(\Omega^{m}\right)}^{2}+\left\|\nabla v_{m}\right\|_{L^{2}\left(\Omega^{m}\right)}^{2} \leq\left\|v_{m}^{*}\right\|_{L^{2}(\Omega)}^{2}+C \frac{1}{A_{m} \lambda}:
$$

by taking into account (5.25) and the strong convergence of $v_{m}^{*}$ to zero in $L^{2}(\Omega)$ we have a contradiction. Then estimate (5.24) is proved and we can repeat the proof of Theorem 5.2 in order to prove that the sequence of the solutions $u_{n}$ [defined in (3.11)] converges to the function $u$ [defined in (2.25)] weakly in $H_{l o c}^{1}(\Omega)$. We show that assumptions (5.21) and (5.22) imply the weak convergence in $L^{2}(\Omega)$. In fact, by (3.13), (5.2) and (5.23),

$$
\begin{aligned}
& \lambda\left\|\nabla u_{n}\right\|_{L^{2}\left(\Omega^{n}\right)}^{2}+c_{n} \sigma_{n} \int_{\Sigma^{n}}\left|\nabla u_{n}\right|^{2} w^{n} d x d y \\
& \quad \leq \int_{\Omega^{n}} f_{n} u_{n} d x d y+C+\frac{|| f_{n} \|_{L^{2}\left(\Sigma^{n}\right)}^{2}}{c_{n} \sigma_{n} \alpha^{n}} c_{n} \sigma_{n} \int_{\Sigma^{n}}\left|\nabla u_{n}\right|^{2} w^{n} d x d y .
\end{aligned}
$$

Hence, by (5.22)

$$
\lambda|| \nabla u_{n} \|_{L^{2}\left(\Omega^{n}\right)}^{2}+c_{n} \sigma_{n} \int_{\Sigma^{n}}\left|\nabla u_{n}\right|^{2} w^{n} d x d y \leq C
$$

and by (3.13)

$$
\left\|u_{n}\right\|_{L^{2}\left(\Sigma^{n}\right)}^{2} \leq \frac{C}{c_{n} \sigma_{n} \alpha^{n}}
$$

From (5.21), (5.24), (5.38) we deduce the uniform boundedness in $L^{2}(\Omega)$. Finally, if we require assumption (5.3), we can show the strong convergence as in the proof of Theorem 5.2 by using (5.38).

Remark 5.3 We note that if

$$
f_{n}=f \quad \text { on } \Omega^{n}, \quad f_{n}=0 \quad \text { on } \widehat{\Omega}^{1} \backslash \Omega^{n}
$$

then assumptions (5.1) and (5.20) are obviously fulfilled; therefore, for the weak convergence in $H_{l o c}^{1}(\Omega)$, no conditions on the vanishing rate of the coefficients $c_{n}$ are required. If we do 
not assume (5.39), we can link the vanishing rate of the sequence $c_{n}$ to the vanishing rate of the $L^{2}$-norms of the data $f_{n}$ in the reinforcement sets $\Sigma^{n}$.

Remark 5.4 We note that the fractal setting gives rise to a peculiar phenomenon. Owing to the tricky geometry, the reinforced domains have to be constructed starting from suitable inner polygonal domains $\Omega^{n}$. Then the functions $u_{n}$ are solutions to equations with reinforced coefficients in a (small) part of the limit domain $\left(\Omega \backslash \Omega^{n}\right)$. Consequently, the reinforced solutions have gradients that are not uniformly bounded in $L^{2}$-norms (in the limit domain), which is in contrast to the strong convergence of the gradients (in $L^{2}$ ) established in [3]. We give simple examples where the assumptions of Theorem 9.3 in [3] are fulfilled but the gradients are not strongly convergent (see Remarks 6.2 and 6.3 following). Moreover, in order to compare Theorem 5.3 with Theorem 9.3 in [3], we note that in our setting we recover the smooth case with $\alpha=4\left(\sigma_{n}=1\right)$; then condition (5.20) - that guarantees the weak convergence in $H_{l o c}^{1}(\Omega)$-is weaker than the assumptions of Theorem 9.3 in [3].

Remark 5.5 If we assume conditions (2.31)-(2.33), then the results of Theorem 5.3 hold for the obstacle problem where

$$
\mathcal{K}_{n}^{\#}=\left\{u \in H_{0}^{1}\left(\Omega, w^{n}\right), \quad u \leq\left(\varphi_{2}\right)_{n}\right\} .
$$

Similar results hold for the two obstacle problems in the assumptions and notation of Remarks 2.4 and 5.2. Moreover, for the two obstacle problems we also obtain strong convergence in $L^{p}(\Omega)$ with $p<+\infty$.

\section{Comments}

In this section we discuss some simple examples. Our first example shows that the results of Theorems 5.1 and 5.2 are sharp.

Example 1 Consider the reinforced obstacle problem in the 1-dimensional case where $\Omega=$ $[-1,1], \Omega^{n}=[-1+\varepsilon, 1-\varepsilon], \widehat{\Omega}^{n}=[-1-\varepsilon, 1+\varepsilon]$ where

$$
\varphi=0, f_{n}=1 \text { in } \widehat{\Omega}^{n}, a_{n}=1 \text { in } \Omega^{n}, a_{n}=\lambda \text { in } \widehat{\Omega}^{n} \backslash \Omega^{n} .
$$

The solution to the reinforced obstacle problem is

$$
u_{n}= \begin{cases}-\frac{x^{2}}{2}+\frac{4 \varepsilon+\lambda(1-\varepsilon)^{2}}{2 \lambda} & \text { in } \Omega^{n} \\ -\frac{x^{2}}{2 \lambda}+\frac{(1+\varepsilon)^{2}}{2 \lambda} & \text { in } \widehat{\Omega}^{n} \backslash \Omega^{n} .\end{cases}
$$

The $L^{2}\left(\Omega \backslash \Omega^{n}\right)$-norms of the derivatives of the functions $u_{n}$ defined in (6.2) are

$$
\left\|u_{n}^{\prime}\right\|_{L^{2}\left(\Omega \backslash \Omega^{n}\right)}^{2}=2 \frac{3 \varepsilon-3 \varepsilon^{2}+\varepsilon^{3}}{3 \lambda^{2}}
$$

and the $L^{2}\left(\Omega \backslash \Omega^{n}\right)$-norms of the functions $u_{n}$ defined in (6.2) are

$$
\left\|u_{n}\right\|_{L^{2}\left(\Omega \backslash \Omega^{n}\right)}^{2}=2 \frac{7 / 3 \varepsilon^{3}+11 / 12 \varepsilon^{4}+2 / 15 \varepsilon^{5}}{\lambda^{2}} .
$$

Remark 6.1 We note that we can not expect weak convergence in the space $H^{1}(\Omega)$. In fact, if we choose $\lambda=\varepsilon$ then the limit problem is the obstacle problem with Robin condition where 


$$
\varphi=0, f=1 \text { in } \Omega, \quad u^{\prime}(1)+1 / 2 u(1)=-u^{\prime}(-1)+1 / 2 u(-1)=0
$$

and the solution is

$$
u=-\frac{x^{2}}{2}+\frac{5}{2}
$$

(see Theorem 5.2) but the sequence of the derivatives of the functions $u_{n}$ is not uniformly bounded in the space $L^{2}(\Omega)$. If we choose $\lambda=(\varepsilon)^{1 / 2}$ then the limit problem is the obstacle problem with Dirichlet condition where

$$
\varphi=0, f=1 \quad \text { in } \Omega, \quad u(1)=u(-1)=0 .
$$

All the assumptions of Theorem 5.1 are fulfilled and the solution is

$$
u=-\frac{x^{2}}{2}+\frac{1}{2}
$$

but the sequence of the derivatives of the functions $u_{n}$ is not strongly convergent in the space $L^{2}(\Omega)$. If we choose $\lambda=(\varepsilon)^{1 / 2+\eta}, \eta \in(0,1 / 2)$ then all the assumptions of Theorem 5.1 are still fulfilled, the limit solution is $(6.8)$ but the sequence of the derivatives of the functions $u_{n}$ is not uniformly bounded in the space $L^{2}(\Omega)$.

Remark 6.2 We discuss the behaviour of $L^{2}(\Omega)$-norms of the functions $u_{n}$ defined in (6.2). Formula (6.4) shows that condition (5.3) does not assure that the sequence of norms in $L^{2}(\Omega)$ of the functions $u_{n}$ is uniformly bounded if the coerciveness assumption (2.14) is not fulfilled (see Theorem 5.2). In fact, if we choose $\lambda=\varepsilon^{2-\eta}, \eta \in(0,1 / 2)$ condition (5.3) is satisfied but the sequence of norms in $L^{2}(\Omega)$ of the functions $u_{n}$ diverges like $\varepsilon^{\eta-\frac{1}{2}}$. Now we discuss the previous example in relation to the semicoercive case. Formula (6.4) shows that conditions (5.21) and (5.22) do not assure that the sequence of norms in $L^{2}(\Omega)$ of the functions $u_{n}$ is uniformly bounded if the sign condition (2.24) is not fulfilled (see Theorem 5.3). If we choose $\lambda=\varepsilon^{5 / 3}$ condition (5.3) is satisfied but the sequence of norms in $L^{2}(\Omega)$ of the functions $u_{n}$ diverges like $\varepsilon^{-1 / 6}$. If instead $\lambda=\varepsilon^{2}$ conditions (5.21) and (5.22) are satisfied but the sequence of norms in $L^{2}(\Omega)$ of the functions $u_{n}$ diverges like $\varepsilon^{-1 / 2}$. Finally we note that with this choice the condition of Remark 1 (page 243) in [3] is satisfied whereas the uniform boundedness of the $L^{2}(\Omega)$-norms (see (9.9) in [3]) fails. We point out that sign condition (2.24) is not fulfilled (see also Remark 2 (page 243) in [3]).

In the following examples, the sign condition (2.24) is satisfied. Example 2 shows that we cannot expect weak convergence in the space $H^{1}(\Omega)$ under condition (5.39) in Theorem 5.3 (see Remark 5.3).

Example 2 Let us consider the obstacle problem in the 1-dimensional case where $\Omega=$ $[-1,1], \varphi_{1}=0$ and $f=\left\{\begin{array}{ll}-2 & \text { if }|x| \leq \frac{1}{2} \\ 1 & \text { if } \frac{1}{2}<|x| \leq 1\end{array}\right.$ with Neumann condition $u^{\prime}(1)=$ $-u^{\prime}(-1)=0:$ the solution is

$$
u= \begin{cases}0 & \text { if }|x| \leq \frac{1}{2} \\ -\frac{x^{2}}{2}+|x|-\frac{3}{8} & \text { if } \frac{1}{2}<|x| \leq 1\end{cases}
$$


Set $\Omega^{n}=[-1+\varepsilon, 1-\varepsilon], \widehat{\Omega}^{n}=[-1-\varepsilon, 1+\varepsilon]$,

$$
f_{n}=\left\{\begin{array}{ll}
-2 & \text { if }|x| \leq \frac{1}{2} \\
1 & \text { if } \frac{1}{2}<|x| \leq 1-\varepsilon \\
0 & \text { if } 1-\varepsilon<|x| \leq 1+\varepsilon
\end{array},\right.
$$

and

$$
a_{n}=1 \text { in } \Omega^{n}, \quad a_{n}=\lambda \text { in } \widehat{\Omega}^{n} \backslash \Omega^{n} .
$$

The reinforced problem has the solution

$$
u_{n}= \begin{cases}0 & \text { if }|x| \leq \frac{1}{2} \\ -\frac{x^{2}}{2}+b|x|+\frac{1}{8}-\frac{b}{2} & \text { if } \frac{1}{2}<|x| \leq 1-\varepsilon \\ \frac{b-1+\varepsilon}{\lambda}|x|-\frac{b-1+\varepsilon}{\lambda}(1+\varepsilon) & \text { if } 1-\varepsilon<|x| \leq 1+\varepsilon\end{cases}
$$

with

$$
b=\frac{16-16 \varepsilon+3 \frac{\lambda}{\varepsilon}+4 \varepsilon \lambda-8 \lambda}{4\left(4+\frac{\lambda}{\varepsilon}-2 \lambda\right)} .
$$

The $L^{2}\left(\Omega \backslash \Omega^{n}\right)$-norms of the derivatives of the functions $u_{n}$ defined in (6.11) are

$$
\left\|u_{n}^{\prime}\right\|_{L^{2}\left(\Omega \backslash \Omega^{n}\right)}^{2}=2 \frac{\varepsilon(b-1+\varepsilon)^{2}}{\lambda^{2}} .
$$

Remark 6.3 It is easy to verify that the sequence of the derivatives of the functions $u_{n}$ is not uniformly bounded in the space $L^{2}(\Omega)$. In fact formula (6.12) shows that if $\frac{\lambda}{\varepsilon} \rightarrow 0$ then $\left\|u_{n}^{\prime}\right\|_{L^{2}\left(\Omega \backslash \Omega^{n}\right)}^{2}$ behaves like $\frac{2}{16^{2} \varepsilon}$. In particular, if we choose $\lambda=\varepsilon^{2}$ then all the conditions of Theorem 9.3 in [3] are satisfied while the uniform boundedness of the $L^{2}(\Omega)$-norms of the gradients [see (9.5)] fails. We think that this discrepancy is due to the fact that the reinforcement goes inside the domain $\Omega$, a choice that is imposed by the tricky geometry of the fractal (see also Remark 5.4).

Example 3 shows that we cannot expect weak convergence in the space $H^{1}(\Omega)$ in the assumptions of Theorem 5.3.

Example 3 Let us consider the obstacle problem of Example 2. In the reinforced problems, we choose the approximating data $f_{n}$ according to Theorem 5.3, the coefficients of the operators being as before. Then

$$
f_{n}= \begin{cases}-2 & \text { if }|x| \leq \frac{1}{2} \\ 1 & \text { if } \frac{1}{2}<|x| \leq 1+\varepsilon\end{cases}
$$

the coefficients are

$$
a_{n}=1 \text { in } \Omega^{n}, a_{n}=\lambda \text { in } \widehat{\Omega}^{n} \backslash \Omega^{n}
$$

and the solution is

$$
u_{n}= \begin{cases}0 & \text { if }|x| \leq \frac{1}{2} \\ -\frac{x^{2}}{2}+b|x|+\frac{1}{8}-\frac{b}{2} & \text { if } \frac{1}{2}<|x| \leq 1-\varepsilon \\ -\frac{x^{2}}{2 \lambda}+\frac{b|x|}{\lambda}-\frac{b(1+\varepsilon)}{\lambda}+\frac{(1+\varepsilon)^{2}}{2 \lambda} & \text { if } 1-\varepsilon<|x| \leq 1+\varepsilon\end{cases}
$$


with

$$
b=\frac{16+3 \frac{\lambda}{\varepsilon}+4 \varepsilon \lambda-8 \lambda}{4\left(4+\frac{\lambda}{\varepsilon}-2 \lambda\right)} .
$$

The $L^{2}\left(\Omega \backslash \Omega^{n}\right)$-norms of the derivatives of the functions $u_{n}$ defined in (6.15) are

$$
\left\|u_{n}^{\prime}\right\|_{L^{2}\left(\Omega \backslash \Omega^{n}\right)}^{2}=2 \frac{\varepsilon(b-1)^{2}+\varepsilon^{2}(b-1)+\varepsilon^{3} 1 / 3}{\lambda^{2}}
$$

The $L^{2}\left(\Omega \backslash \Omega^{n}\right)$-norms of the functions $u_{n}$ defined in (6.15) are

$$
\left\|u_{n}\right\|_{L^{2}\left(\Omega \backslash \Omega^{n}\right)}^{2}=\frac{7 / 3 \varepsilon^{3}(b-1)^{2}+11 / 3 \varepsilon^{4}(1-b)+8 / 15 \varepsilon^{5}}{2 \lambda^{2}} .
$$

Remark 6.4 It is easy to verify that the sequence of the derivatives of the functions $u_{n}$ is not uniformly bounded in the space $L^{2}(\Omega)$. In fact, formula (6.16) shows that if $\frac{\lambda}{\varepsilon} \rightarrow 0$ then $\left\|u_{n}^{\prime}\right\|_{L^{2}\left(\Omega \backslash \Omega^{n}\right)}^{2}$ behaves like $\frac{2}{16^{2} \varepsilon}-\frac{2 \varepsilon}{16 \lambda}+\frac{2 \varepsilon^{3}}{3 \lambda^{2}}$. If we choose $\lambda=\varepsilon^{5 / 3}$, condition (5.20) is satisfied but the sequence of norms in $L^{2}(\Omega)$ of the derivatives of the functions $u_{n}$ diverges. As regards the behaviour of the functions, formula (6.17) shows that $\left\|u_{n}\right\|_{L^{2}\left(\Omega \backslash \Omega^{n}\right)}^{2}$ behaves like $\max \left(\frac{\varepsilon^{5}}{\lambda^{2}}, \frac{\varepsilon^{3}}{\lambda}\right)$. If we choose $\lambda=\varepsilon^{5 / 2+\eta}, 0<\eta<\frac{1}{2}$, then condition (5.21) does not hold [only condition (5.22) is satisfied] and the sequence of $L^{2}(\Omega)$-norms of the functions $u_{n}$ diverges like $\varepsilon^{-\eta}$.

\section{Interior reinforcement}

In [14] we established homogenization results for an insulating fractal surface $S$ of Koch type which is approximated by 3-dimensional insulating layers with both vanishing conductivity and thickness. Also in this case we consider the corresponding obstacle problems.

We consider a 3-dimensional Euclidean domain $Q$ containing a fractal subset $S$, the layer. Our basic model refers to the geometry illustrated in Fig. 1. Here the layer is of the type

$$
S=K \times I,
$$

where $K$ is the so-called Koch type curve defined in Sect. 2 and

$$
Q=\mathcal{O} \times(0,1)=(0,1) \times\left(-\frac{1}{2}, \frac{1}{2}\right) \times(0,1) ;
$$

every point $P \in Q$ has coordinates $\left(x_{1}, x_{2}, y\right)$ and the boundary of $S$ belongs to the boundary of $Q$. We note that the layer $S$ is a $d$-set in $\mathbb{R}^{3}$ with respect to the measure $d \mu d y$ with $d=d_{S}=d_{f}+1$ (see [18]). The fractal layer $S$ divides the domain $Q$ in two adjacent subdomains $Q_{i}, i=1,2$, where $Q_{1}$ denotes the domain above the layer $S$.

We denote by $\Sigma^{0}$ the open set condition triangle of vertices $A=(0,0), B=(1,0)$ and $C=(1 / 2, b / 2)$ where $b=\tan \left(\frac{\theta}{2}\right)$ and by $K^{0}$ the line segment that has as endpoints $A$ and $B$.

For every $n$, we define the (open) polygonal fiber $\Sigma^{n}$ in the cross-section

$$
\Sigma^{n}=\bigcup_{i \mid n} \Sigma^{i \mid n} \quad \text { where } \Sigma^{i \mid n}=\psi_{i \mid n}\left(\Sigma^{0}\right)
$$


Fig. 1 Basic model

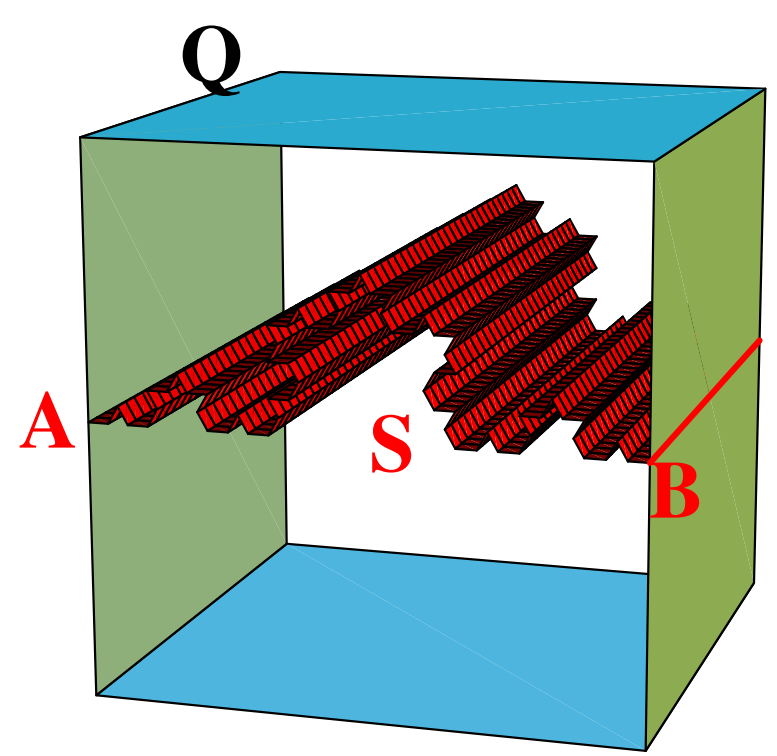

and the auxiliary curve

$$
\check{K}^{n}=\bigcup_{i \mid n} \check{K}^{i \mid n} \quad \text { where } \check{K}^{i \mid n}=\partial \Sigma^{i \mid n} \backslash \stackrel{\circ}{K^{i \mid n}}, \quad K^{i \mid n}=\psi_{i \mid n}\left(K^{0}\right) .
$$

We set $\Gamma^{0}=\Sigma^{0} \times \stackrel{\circ}{I}, \Gamma^{i \mid n}=\Sigma^{i \mid n} \times \stackrel{\circ}{I}, \Gamma^{n}=\Sigma^{n} \times \stackrel{\circ}{I}, S^{0}=K^{0} \times I, S^{i \mid n}=K^{i \mid n} \times I$, $S^{n}=K^{n} \times I, \check{K}^{0}=\partial \Sigma^{0} \backslash \stackrel{\circ}{K^{0}}, \mathcal{G}^{0}=\check{K}^{0} \times I, \mathcal{G}^{i \mid n}=\check{K}^{i \mid n} \times I$ and $\mathcal{G}^{n}=\check{K}^{n} \times I$. The polyhedral surfaces $S^{n}$ and $\mathcal{G}^{n}$ divide the domain $Q$ into three subdomains. We denote by $\hat{Q}_{n}^{2}$ the domain below $S^{n}$ and by $\hat{Q}_{n}^{1}$ the domain above $\mathcal{G}^{n}$, that is

$$
Q=\overbrace{\hat{Q}_{n}^{1} \cup \bar{\Gamma}^{n} \cup \hat{Q}_{n}^{2}}^{\circ} .
$$

We note that if $P=(x, y) \in \Gamma^{n}$, then $x \in \Sigma^{n}$. Each $x \in \Sigma^{n}$ belongs to a segment of endpoints $X$ and $X^{\perp}$ where $X \in \check{K}^{i \mid n}$ and $X^{\perp}$ is the orthogonal projection of $X$ on $K^{i \mid n}$ for some index $i \mid n$. By $\left|X-X^{\perp}\right|$ we denote the (Euclidean) distance between $X$ and $X^{\perp}$ (in $\mathbb{R}^{2}$ ).

In the domain $Q$, for any $n$, we define a weight $w^{n}$ as follows. Let $P=(x, y) \in Q$

$$
w^{n}(P)=w^{n}(x)= \begin{cases}\frac{3\left|X-X^{\perp}\right|}{3+b^{2}} & \text { if }(x, y) \in \Gamma^{i \mid n} \\ 1 & \text { if }(x, y) \notin \Gamma^{n} .\end{cases}
$$

Associated with the weight $w^{n}$, we consider the Sobolev spaces $H^{1}\left(Q ; w^{n}\right)$ and $H_{0}^{1}\left(Q ; w^{n}\right)$, defined as the completion of $C^{\infty}(\bar{Q})$ and $C_{0}^{\infty}(Q)$, respectively, in the norm

$$
\|u\|_{H^{1}\left(Q ; w^{n}\right)}=\left\{\int_{Q} u^{2} d x d y+\int_{Q}|\nabla u|^{2} w^{n} d x d y\right\}^{\frac{1}{2}} .
$$

We consider the sequence of weighted energy functionals in $L^{2}(Q)$

$$
a_{n}(u, v):=\int_{Q} \sum_{i, j=1}^{3} a_{i j}^{n} \frac{\partial u}{\partial x_{i}} \frac{\partial v}{\partial x_{j}} d x d y
$$


where we define the coefficients

$$
a_{i j}^{n}(x, y)= \begin{cases}\sigma_{n} c_{n} \delta_{i j} w^{n}(x) & \text { if }(x, y) \in \Gamma^{n} \\ a_{i j} & \text { if }(x, y) \in Q \backslash \Gamma^{n}\end{cases}
$$

where

$$
c_{n}>0, \quad \sigma_{n}=\frac{\alpha^{n}}{4^{n}}
$$

and the coefficients $a_{i j}$ with $1 \leq i, j \leq 3$ satisfy (2.6) and

$$
\lambda|\xi|^{2} \leq \sum_{i, j=1}^{3} a_{i j} \xi_{i} \xi_{j} \leq \Lambda|\xi|^{2}
$$

for $\Lambda \geq \lambda>0$.

We define the set

$$
\mathcal{V}(Q)=\left\{g \in L^{2}(Q): \exists g_{j}^{*} \in \operatorname{Lip}\left(\bar{Q}_{j}\right):\left.g_{j}^{*}\right|_{Q_{j}}=\left.g\right|_{Q_{j}}\right\} .
$$

From now on, when it does not create ambiguity, we drop the superscript $*$ and we simply write $u_{j}, g_{j}$ and similar expressions.

We recall that $\Sigma^{0}$ is the open set condition triangle of vertices $A=(0,0), B=(1,0)$ and $C=(1 / 2, b / 2)$ where $b=\tan \left(\frac{\theta}{2}\right)$, we then divide $\Sigma^{0}$ into two triangles $\mathcal{T}_{h}, h=1,2$ with vertices $A, H, C$ and $H, B, C$ respectively, where $H=(1 / 2,0)$.

We denote by $G$ the operator from $\mathcal{V}(Q)$ to $H^{1}\left(\Gamma_{0}\right)$ defined as follows: for $\left(\xi_{1}, \xi_{2}, y\right) \in \bar{\Gamma}_{0}$

$$
\begin{aligned}
& G(g)\left(\xi_{1}, \xi_{2}, y\right) \\
& = \begin{cases}g_{2}(0,0, y) & \text { if }\left(\xi_{1}, \xi_{2}\right)=(0,0) \\
\frac{\xi_{2}}{b \xi_{1}} g_{1}\left(\xi_{1}, b \xi_{1}, y\right)+\left(1-\frac{\xi_{2}}{b \xi_{1}}\right) g_{2}\left(\xi_{1}, 0, y\right) & \text { if }\left(\xi_{1}, \xi_{2}\right) \in \overline{\mathcal{T}}_{1} \backslash\{A\} \\
\frac{\xi_{2}}{b\left(1-\xi_{1}\right)} g_{1}\left(\xi_{1}, b\left(1-\xi_{1}\right), y\right)+\left(1-\frac{\xi_{2}}{b\left(1-\xi_{1}\right)}\right) g_{2}\left(\xi_{1}, 0, y\right) & \text { if }\left(\xi_{1}, \xi_{2}\right) \in \overline{\mathcal{T}}_{2} \backslash\{B\} \\
g_{2}(1,0, y) & \text { if }\left(\xi_{1}, \xi_{2}\right)=(1,0) .\end{cases}
\end{aligned}
$$

For every $u \in \mathcal{V}(Q)$ and $n$ we define in $\overline{\Gamma^{i \mid n}}$ the function $v_{i \mid n}$

$$
v_{i \mid n}(x, y)=G\left(u \circ \Psi_{i \mid n}\right) \circ \Psi_{i \mid n}^{-1}(x, y) \quad \text { if }(x, y) \in \overline{\Gamma^{i \mid n}}
$$

where $\Psi_{i \mid n}\left(\xi_{1}, \xi_{2}, y\right)=\left(\psi_{i \mid n}\left(\xi_{1}, \xi_{2}\right), y\right)$. For every $u \in \mathcal{V}(Q)$ and $n$ we define

$$
v_{n}(x, y)= \begin{cases}u_{j}(x, y) & \text { if }(x, y) \in \hat{Q}_{j}^{n}, j=1,2 \\ v_{i \mid n}(x, y) & \text { if }(x, y) \in \overline{\Gamma^{i \mid n}} .\end{cases}
$$

We assume the following condition on the obstacle

$$
\varphi_{1} \in C^{1}(\bar{Q}), \varphi_{1} \leq 0 \quad \text { on } \partial Q,
$$

and we first study the coercive case, that is, we choose the convex sets

$$
\mathcal{K}_{\infty}=\left\{u \in H_{0}^{1}(Q), u \geq \varphi_{1}\right\}
$$

and

$$
\mathcal{K}=\left\{u \in D_{0}(Q), u \geq \varphi_{1}\right\}
$$

where $D_{0}(Q)=\left\{u \in L^{2}(Q): u_{j}:=\left.u\right|_{Q_{j}} \in H^{1}\left(Q_{j}\right), u_{j}=0\right.$ on $\left.\partial Q_{j} \backslash S\right\}$. 
We define the limit forms as follows

$$
\begin{aligned}
a_{c_{0}}(u, v)= & \int_{Q_{1}} \sum_{i, j=1}^{3} a_{i j} \frac{\partial u}{\partial x_{i}} \frac{\partial v}{\partial x_{j}} d x d y+\int_{Q_{2}} \sum_{i, j=1}^{3} a_{i j} \frac{\partial u}{\partial x_{i}} \frac{\partial v}{\partial x_{j}} d x d y \\
& +\int_{S} c_{0}\left(u_{1}-u_{2}\right)\left(v_{1}-v_{2}\right) d \mu d y
\end{aligned}
$$

and

$$
a_{\infty}(u, v)=\int_{Q} \sum_{i, j=1}^{3} a_{i j} \frac{\partial u}{\partial x_{i}} \frac{\partial v}{\partial x_{j}} d x d y .
$$

We construct a suitable obstacle $\left(\varphi_{1}\right)_{n}$ according to the formula (7.14) and we consider

$$
\mathcal{K}_{n}=\left\{u \in H_{0}^{1}\left(Q ; w^{n}\right), u \geq\left(\varphi_{1}\right)_{n}\right\} .
$$

Before stating our results in this framework, we specify, in this geometry, an inequality Poincare type where, as previously in Sect. 3, the relevant fact is that the constant $C_{P}$ is independent of $n$. The proof is similar to the proof of Theorem 7.4 (following), hence we skip it (see also Theorem 6.1 in [13]).

Theorem 7.1 For any function $u \in H^{1}\left(Q ; w^{n}\right)$ the following estimate holds

$$
\|u\|_{L^{2}\left(\Gamma^{n}\right)}^{2} \leq \alpha^{-n}\left(\int_{\Gamma^{n}}|\nabla u|^{2} w^{n} d x d y+\int_{S^{n}} u^{2} d s d y\right) .
$$

Moreover, there exists a constant $C_{P}$ independent of $n$, such that,

$$
\|u\|_{L^{2}(Q)} \leq C_{P}\left(\|\nabla u\|_{L^{2}\left(Q \backslash \Gamma^{n}\right)}^{2}+\alpha^{-n} \int_{\Gamma^{n}}|\nabla u|^{2} w^{n} d x d y\right)^{1 / 2}
$$

for all $u \in H_{0}^{1}\left(Q ; w^{n}\right)$.

Taking into account Poincaré inequality (7.19) and the classical Poincaré inequality (see e.g. [23]), we can prove existence and uniqueness results. More precisely

Proposition 7.1 For any $f_{n} \in L^{2}(Q)$, there exists one and only one solution $u_{n}$ to the following problem

$$
\left\{\begin{array}{l}
\text { find } u_{n} \in \mathcal{K}_{n} \text { such that } \\
a_{n}\left(u_{n}, v-u_{n}\right) \geq \int_{Q} f_{n}\left(v-u_{n}\right) d x d y \quad \forall v \in \mathcal{K}_{n}
\end{array}\right.
$$

where $a_{n}$ is defined in (7.7). For any $f \in L^{2}(Q)$, there exists one and only one solution $u$ to the following problem

$$
\left\{\begin{array}{l}
\text { find } u \in \mathcal{K}_{\infty} \text { such that } \\
a_{\infty}(u, v-u) \geq \int_{Q} f(v-u) d x d y \quad \forall v \in \mathcal{K}_{\infty}
\end{array}\right.
$$

where $a_{\infty}$ is defined in (7.17). Moreover, for any $f \in L^{2}(Q), c_{0} \geq 0$, there exists one and only one solution $u$ to the following problem

$$
\left\{\begin{array}{l}
\text { find } u \in \mathcal{K} \text { such that } \\
a_{c_{0}}(u, v-u) \geq \int_{Q} f(v-u) d x d y \quad \forall v \in \mathcal{K}
\end{array}\right.
$$

where $a_{c_{0}}$ is defined in (7.16). 
We assume

$$
f_{n}, f \in L^{2}(Q) \text {, and } f_{n} \rightarrow f \text { in } L^{2}(Q) ;
$$

by taking into account the results of Theorems 1.2 and 1.1 of [14], we obtain the following results that we can prove by proceeding as in Sect. 5 .

Theorem 7.2 Let us assume (4.21) and (7.23). Then the sequence of the solutions $u_{n}[$ defined in (7.20)] converges to the function $u$ [defined in (7.21)] weakly in $H_{l o c}^{1}\left(Q_{i}\right), i=1,2$, and strongly in $L^{2}(Q)$.

Theorem 7.3 Let us assume (4.6), (5.21), and (7.23). Then the sequence of the solutions $u_{n}\left[\right.$ defined in (7.20)] converges to the function $u$ [defined in (7.22)] weakly in $H_{l o c}^{1}\left(Q_{i}\right)$, $i=1,2$, and weakly in $L^{2}(Q)$. Moreover, if we assume condition (5.3), then the sequence of the solutions $u_{n}$ converges to the function $u$ strongly in $L^{2}(Q)$.

We will now consider the semi-coercive case. Let

$$
\mathcal{F}_{n}=\left\{u \in H^{1}\left(Q ; w^{n}\right), u \geq\left(\varphi_{1}\right)_{n}\right\}
$$

and

$$
\mathcal{F}=\left\{u \in D(Q), u \geq \varphi_{1}\right\}
$$

where $D(Q)=\left\{u \in L^{2}(Q): u_{j}:=\left.u\right|_{Q_{j}} \in H^{1}\left(Q_{j}\right)\right\}$, the obstacle $\varphi_{1} \in C^{1}(\bar{Q})$ and the obstacle $\left(\varphi_{1}\right)_{n}$ is as in (7.14). We recall that in the semi-coercive case, we have $c_{0}=0$, hence we assume suitable conditions on the data $f, f_{n}$ and on the convex [see (2.23) and (2.24)]. More precisely, we assume

$$
\begin{aligned}
& 0 \in \mathcal{F}, \\
& \int_{Q_{i}} f d x d y<0 \quad i=1,2 \\
& \int_{Q} f_{n} d x d y<0 .
\end{aligned}
$$

As in [22] and [32], see also the proof of Theorem 2.3, we can show the following proposition

Proposition 7.2 Assume $f_{n} \in L^{2}(Q)$, (7.24) and (7.26). Then there exists one and only one solution $u_{n}$ to the following problem

$$
\left\{\begin{array}{l}
\text { find } u_{n} \in \mathcal{F}_{n} \text { such that } \\
a_{n}\left(u_{n}, v-u_{n}\right) \geq \int_{Q} f_{n}\left(v-u_{n}\right) d x d y \quad \forall v \in \mathcal{F}_{n}
\end{array}\right.
$$

where $a_{n}$ is defined in (7.7). Assume $f \in L^{2}(Q)$, (7.24) and (7.25). Then there exists one and only one solution $u$ to the following problem

$$
\left\{\begin{array}{l}
\text { find } u \in \mathcal{F} \text { such that } \\
a(u, v-u) \geq \int_{Q} f(v-u) d x d y \quad \forall v \in \mathcal{F}
\end{array}\right.
$$

where

$$
a(u, v)=\int_{Q_{1}} \sum_{i, j=1}^{3} a_{i j} \frac{\partial u}{\partial x_{i}} \frac{\partial v}{\partial x_{j}} d x d y+\int_{Q_{2}} \sum_{i, j=1}^{3} a_{i j} \frac{\partial u}{\partial x_{i}} \frac{\partial v}{\partial x_{j}} d x d y
$$


Before stating our results in this framework, we specify, in this geometry, an inequality of Poincare type, where the relevant fact is that the constant $C_{P}$ is independent of $n$.

Theorem 7.4 For any function $u \in H^{1}\left(Q ; w^{n}\right)$, the following estimate holds

$$
\|u\|_{L^{2}\left(\Gamma^{n}\right)}^{2} \leq \alpha^{-n}\left(\int_{\Gamma^{n}}|\nabla u|^{2} w^{n} d x d y+\int_{S^{n}} u^{2} d s d y\right) .
$$

Moreover, there exists a constant $C_{P}$ independent of $n$, such that,

$$
\|u\|_{L^{2}(Q)} \leq C_{P}\left(\|\nabla u\|_{L^{2}\left(Q \backslash \Gamma^{n}\right)}^{2}+\sigma_{n} \int_{\Gamma^{n}}|\nabla u|^{2} w^{n} d x d y+\sigma_{n} \int_{S^{n}} u^{2} d s d y\right)^{1 / 2}
$$

for all $u \in H^{1}\left(Q ; w^{n}\right)$.

Proof We start by proving estimate (7.30). For every $n$, we split the corresponding integrals

$$
\int_{\Sigma^{n}} u^{2} d x=\sum_{i \mid n} \int_{\Sigma^{i \mid n}} u^{2} d x
$$

It suffices to give the proof for $\Sigma^{i \mid n}$, as the other integrals can be evaluated similarly. By the change of coordinates $x=\left(x_{1}, x_{2}\right)=\psi_{i \mid n}\left(\xi_{1}, \xi_{2}\right)$, with $\left(\xi_{1}, \xi_{2}\right) \in \Sigma^{0}$, we define

$$
g\left(\xi_{1}, \xi_{2}\right)=\left(u \circ \psi_{i \mid n}\right)\left(\xi_{1}, \xi_{2}\right)
$$

and we have

$$
\begin{aligned}
\int_{\Sigma^{i \mid n}} u^{2} d x & =\alpha^{-2 n}\left\{\int_{0}^{1 / 2} d \xi_{1} \int_{0}^{b \xi_{1}} g^{2}\left(\xi_{1}, \xi_{2}\right) d \xi_{2}+\int_{1 / 2}^{1} d \xi_{1} \int_{0}^{b\left(1-\xi_{1}\right)} g^{2}\left(\xi_{1}, \xi_{2}\right) d \xi_{2}\right\} \\
& :=\alpha^{-2 n}\left\{I_{1}+I_{2}\right\} .
\end{aligned}
$$

As

$$
g^{2}\left(\xi_{1}, \xi_{2}\right) \leq 2\left(\left(g\left(\xi_{1}, \xi_{2}\right)-g\left(\xi_{1}, 0\right)\right)^{2}+g^{2}\left(\xi_{1}, 0\right)\right)
$$

then

$$
I_{1} \leq 2\left\{\frac{1}{2} \int_{0}^{1 / 2} d \xi_{1} b^{2} \xi_{1}^{2} \int_{0}^{b \xi_{1}}|\nabla g|^{2} d \xi_{2}+\frac{b}{2} \int_{0}^{1 / 2} g^{2}\left(\xi_{1}, 0\right) d \xi_{1}\right\} .
$$

Analogously

$$
I_{2} \leq 2\left\{\frac{1}{2} \int_{1 / 2}^{1} d \xi_{1} b^{2}\left(1-\xi_{1}\right)^{2} \int_{0}^{b\left(1-\xi_{1}\right)}|\nabla g|^{2} d \xi_{2}+\frac{b}{2} \int_{1 / 2}^{1} g^{2}\left(\xi_{1}, 0\right) d \xi_{1}\right\} .
$$

Then by definition of the weight [see (7.5)]

$$
\alpha^{-2 n}\left\{I_{1}+I_{2}\right\} \leq 2 \alpha^{-2 n}\left\{\frac{b\left(3+b^{2}\right)}{12} \int_{\Sigma^{0}}|\nabla g|^{2} w^{0}\left(\xi_{1}, \xi_{2}\right) d \xi_{1} d \xi_{2}+\frac{b}{2} \int_{K^{0}} g^{2}\left(\xi_{1}, 0\right) d \xi_{1}\right\} .
$$

By the change of coordinates $\left(\xi_{1}, \xi_{2}\right)=\psi_{i \mid n}^{-1}\left(x_{1}, x_{2}\right)$, and taking into account that $\alpha^{-n} w^{0}\left(\psi_{i \mid n}^{-1}\left(x_{1}, x_{2}\right)\right)=w^{n}\left(x_{1}, x_{2}\right)$ we derive 


$$
\int_{\Sigma^{i \mid n}} u^{2} d x \leq \alpha^{-n}\left(\int_{\Sigma^{i \mid n}}|\nabla u|^{2} w^{n} d x+\int_{K^{i \mid n}} u^{2} d s\right) .
$$

By summing up on $i \mid n$ and integrating on the interval $I$ we conclude the proof of (7.30).

Now we prove statement (7.31). Suppose (7.31) is false: then, there exists an increasing sequence of indices $n_{m} \in \mathbb{N}$ and there exists a sequence of functions $u_{m} \in H^{1}\left(Q ; w^{n_{m}}\right)$, such that, for every $m \in \mathbb{N}$,

$$
\left\|u_{m}\right\|_{L^{2}(Q)}^{2}>m^{2}\left(\left\|\nabla u_{m}\right\|_{L^{2}\left(Q \backslash \Gamma^{n_{m}}\right)}^{2}+\sigma_{n_{m}} \int_{\Gamma^{n_{m}}}\left|\nabla u_{m}\right|^{2} w^{n_{m}} d x d y+\sigma_{n_{m}} \int_{S^{n_{m}}} u_{m}^{2} d s d y\right) .
$$

In fact, if such a sequence of indices does not exist we can repeat, for a fixed $\bar{n}$, the argument below to obtain a contradiction again. Set

$$
v_{m}:=\frac{u_{m}}{\left\|u_{m}\right\|_{L^{2}(Q)}} .
$$

Therefore, there exist a function $v^{*}$ in $L^{2}(Q)$ and a subsequence (still denoted by $v_{m}$ ) that converges to $v^{*}$ weakly in $L^{2}(Q)$. From (7.38), we obtain

$$
\left\|\nabla v_{m}\right\|_{L^{2}\left(Q \backslash \Gamma^{n_{m}}\right)}^{2}+\sigma_{n_{m}} \int_{\Gamma^{n_{m}}}\left|\nabla v_{m}\right|^{2} w^{n_{m}} d x d y+\sigma_{n_{m}} \int_{S^{n_{m}}} v_{m}^{2} d s d y<\frac{1}{m^{2}}
$$

and, in particular,

$$
\left\|v_{m}\right\|_{H^{1}\left(Q \backslash \Gamma^{n_{m}}\right)}^{2} \leqslant 1+\frac{1}{m^{2}} .
$$

For $i=1,2$, we consider the restriction to $\hat{Q}_{i}^{n}$ and we denote by $v_{m, i}^{*}$ the extension to $Q$ and we have

$$
\left\|v_{m, i}^{*}\right\|_{H^{1}(Q)} \leq C
$$

with $C$ independent of $n$ and $m$ (see Theorem 5.7 in [9]).

Then, there exist a function $v_{1}^{*}$ in $H^{1}(Q)$ and a subsequence (still denoted by $v_{m, 1}^{*}$ ) that converges to $v_{1}^{*}$ weakly in $H^{1}(Q)$ (and strongly in $H^{s}(Q)$ for $\left.0 \leq s<1\right)$. Analogously, there exist a function $v_{2}^{*}$ in $H^{1}(Q)$ and a subsequence (still denoted by $v_{m, 2}^{*}$ ) that converges to $v_{2}^{*}$ weakly in $H^{1}(Q)$ (and strongly in $H^{s}(Q)$ for $0 \leq s<1$ ). We observe that $\left.v_{i}^{*}\right|_{Q_{i}}=\left.v^{*}\right|_{Q_{i}}$. We fix $n_{0}$ : we deduce

$$
\left\|\nabla v_{i}^{*}\right\|_{L^{2}\left(\hat{Q}_{i}^{n_{0}}\right)} \leq \liminf _{m}\left\|\left.\nabla v_{m}\right|_{\hat{Q}_{i}^{n_{0}}}\right\|_{L^{2}\left(\hat{Q}_{i}^{n_{0}}\right)}=0
$$

i.e. the function $v_{i}^{*}$ is constant on $\hat{Q}_{i}^{n_{0}}$ and we denote this constant by $k_{i}$; passing to the limit in $n_{0}$, we obtain $v_{i}^{*}=k_{i}$ in $Q_{i}$. From (7.39) we obtain that

$$
k_{2}^{2}=\int_{S}\left(v_{2}^{*}\right)^{2} d \mu d y=\lim _{m} \sigma_{n_{m}} \int_{S^{n_{m}}} v_{m}^{2} d s d y=0 .
$$

We prove that $k_{1}=0$ too. We first prove that

$$
\begin{gathered}
\sigma_{n_{m}}\left|\int_{S^{n_{m}}} v_{m, 2}^{*} d s d y-\frac{1}{\sqrt{1+b^{2}}} \int_{\mathcal{G}^{n_{m}}} v_{m, 1}^{*} d s d y\right| \\
\leq\left(\frac{\left(3+b^{2}\right)}{3} \sigma_{n_{m}} \int_{\Gamma^{n_{m}}}\left|\nabla v_{m}\right|^{2} w^{n_{m}} d x d y\right)^{\frac{1}{2}} .
\end{gathered}
$$


In fact by Hölder inequality we obtain

$$
\begin{aligned}
& \left|\int_{K^{n_{m}}} v_{m, 2}^{*} d s-\frac{1}{\sqrt{1+b^{2}}} \int_{\check{K}^{n_{m}}} v_{m, 1}^{*} d s\right| \\
& \quad=\left|\sum_{i \mid n_{m}}\left\{\int_{K^{i \mid n_{m}}} v_{m} d s-\frac{1}{\sqrt{1+b^{2}}} \int_{\check{K}^{i \mid n_{m}}} v_{m} d s\right\}\right| \\
& \quad \leq 4^{\frac{n_{m}}{2}}\left\{\sum_{i \mid n_{m}}\left|\int_{K^{i \mid n_{m}}} v_{m} d s-\frac{1}{\sqrt{1+b^{2}}} \int_{\check{K}^{i \mid n_{m}}} v_{m} d s\right|^{2}\right\}^{\frac{1}{2}} .
\end{aligned}
$$

By the change of coordinates $x=\left(x_{1}, x_{2}\right)=\psi_{i \mid n_{m}}\left(\xi_{1}, \xi_{2}\right)$, we have

$$
\begin{aligned}
\mid & \int_{K^{i \mid n_{m}}} v_{m} d s-\left.\frac{1}{\sqrt{1+b^{2}}} \int_{\check{K}^{i \mid n_{m}}} v_{m} d s\right|^{2} \\
= & \alpha^{-2 n_{m}} \mid \int_{0}^{1} v_{m}\left(\psi_{i \mid n}\left(\xi_{1}, 0\right)\right) d \xi_{1}-\int_{0}^{1 / 2} v_{m}\left(\psi_{i \mid n_{m}}\left(\xi_{1}, b \xi_{1}\right)\right) d \xi_{1} \\
& -\int_{1 / 2}^{1} v_{m}\left(\left.\psi_{i \mid n_{m}}\left(\xi_{1}, b\left(1-\xi_{1}\right)\right) d \xi_{1}\right|^{2}\right. \\
= & \alpha^{-2 n_{m}} \mid \int_{0}^{1 / 2}\left(v_{m}\left(\psi_{i \mid n}\left(\xi_{1}, 0\right)\right)-v_{m}\left(\psi_{i \mid n_{m}}\left(\xi_{1}, b \xi_{1}\right)\right)\right) d \xi_{1} \\
& +\int_{1 / 2}^{1}\left(v_{m}\left(\psi_{i \mid n}\left(\xi_{1}, 0\right)\right)-\left.v_{m}\left(\psi_{i \mid n_{m}}\left(\xi_{1}, b\left(1-\xi_{1}\right)\right)\right) d \xi_{1}\right|^{2}\right. \\
:= & \alpha^{-2 n_{m}}\left|X_{1}+X_{2}\right|^{2} .
\end{aligned}
$$

We proceed as previously in proving estimate (7.30) and we obtain [see definition of the weight (7.5)]

$$
\begin{aligned}
\mid X_{1} & +\left.X_{2}\right|^{2} \leq 2\left(\left|X_{1}\right|^{2}+\left|X_{2}\right|^{2}\right) \\
\leq & 2 \frac{1}{2} \frac{\left(3+b^{2}\right)}{3}\left(\int_{0}^{1 / 2} d \xi_{1} \int_{0}^{b \xi_{1}}\left|\nabla\left(v_{m} \circ \psi_{i \mid n_{m}}\right)\right|^{2} w^{0}\left(\xi_{1}, \xi_{2}\right) d \xi_{2}\right. \\
& \left.+\int_{1 / 2}^{1} d \xi_{1} \int_{0}^{b\left(1-\xi_{1}\right)}\left|\nabla\left(v_{m} \circ \psi_{i \mid n_{m}}\right)\right|^{2} w^{0}\left(\xi_{1}, \xi_{2}\right) d \xi_{2}\right) \\
= & \left.\frac{\left(3+b^{2}\right)}{3} \int_{\Sigma^{0}} \nabla\left(v_{m} \circ \psi_{i \mid n_{m}}\right)\right|^{2} w^{0}\left(\xi_{1}, \xi_{2}\right) d \xi_{2} .
\end{aligned}
$$

By the change of coordinates $\left(\xi_{1}, \xi_{2}\right)=\psi_{i \mid n_{m}}^{-1}\left(x_{1}, x_{2}\right)$, and taking into account that $\alpha^{-n_{m}} w^{0}\left(\psi_{i \mid n_{m}}^{-1}\left(x_{1}, x_{2}\right)\right)=w^{n_{m}}\left(x_{1}, x_{2}\right)$ we derive

$$
\left|\int_{K^{i \mid n_{m}}} v_{m} d s-\frac{1}{\sqrt{1+b^{2}}} \int_{\check{K}^{i \mid n_{m}}} v_{m} d s\right|^{2} \leq \frac{3+b^{2}}{3} \alpha^{-n_{m}} \int_{\Sigma^{i \mid n_{m}}}\left|\nabla v_{m}\right|^{2} w^{n_{m}} d x .
$$

By summing up on $i \mid n_{m}$ and integrating on the interval $I$, we conclude the proof of (7.42). From (7.42), by using (7.39), we obtain

$$
\sigma_{n_{m}}\left|\int_{S^{n_{m}}} v_{m, 2}^{*} d s d y-\frac{1}{\sqrt{1+b^{2}}} \int_{\mathcal{G}^{n_{m}}} v_{m, 1}^{*} d s d y\right| \leq \sqrt{\frac{3+b^{2}}{3}} \frac{1}{m} .
$$


Passing to the limit in (7.45) as $m \rightarrow 0$ we obtain $k_{1}=0$. By (7.30) we deduce

$$
\left\|v_{m}\right\|_{L^{2}\left(\Gamma^{n_{m}}\right)}^{2} \leq \alpha^{-n_{m}}\left(\int_{\Gamma^{n_{m}}}\left|\nabla v_{m}\right|^{2} w^{n_{m}} d x d y+\int_{S^{n_{m}}} v_{m}^{2} d s d y\right) \leq \frac{1}{m^{2}}
$$

therefore, as

$$
\begin{aligned}
1 & =\left\|v_{m}\right\|_{L^{2}(Q)}^{2}=\left\|v_{m}\right\|_{L^{2}\left(\hat{Q}_{1}^{n_{m}}\right)}^{2}+\left\|v_{m}\right\|_{L^{2}\left(\hat{Q}_{2}^{n_{m}}\right)}^{2}+\left\|v_{m}\right\|_{L^{2}\left(\Gamma^{n_{m}}\right)}^{2} \\
& \leq\left\|v_{m, 1}^{*}\right\|_{L^{2}\left(Q_{1}\right)}^{2}+\left\|v_{m, 2}^{*}\right\|_{L^{2}\left(Q_{2}\right)}^{2}+\frac{1}{m^{2}}
\end{aligned}
$$

passing to the limit, we obtain a contradiction.

Theorem 7.5 Let us assume conditions (4.6) with $c_{0}=0$, (7.23)-(7.26), and,

$$
\frac{\left\|f_{n}\right\|_{L^{2}\left(\Gamma^{n}\right)}^{2}}{c_{n} \sigma_{n} \alpha^{n}} \leq c^{*}
$$

with $c^{*}>0$. Then the sequence of the solutions $u_{n}$ [defined in (7.27)] converges to the function $u$ [defined in (7.28)] weakly in $H_{l o c}^{1}\left(Q_{i}\right), i=1$, 2. If assumption (5.21) holds and thus

$$
\frac{\left\|f_{n}\right\|_{L^{2}\left(\Gamma^{n}\right)}^{2}}{c_{n} \sigma_{n} \alpha^{n}} \rightarrow 0
$$

then the solutions $u_{n}$ converge weakly in $L^{2}(Q)$. Moreover, if assumption (5.3) holds then the solutions $u_{n}$ converge strongly in $L^{2}(Q)$.

Remark 7.1 We note that if

$$
f_{n}=f \text { on } \hat{Q}_{i}^{n}, \quad i=1,2 \text { and } f_{n}=0 \text { on } \Gamma^{n}
$$

then assumptions (7.23) and (7.47) are obviously fulfilled; hence, we have weak convergence in $H_{l o c}^{1}\left(Q_{i}\right)$ without assuming any condition on the vanishing rate of the coefficients $c_{n}$. Alternatively, we can link the vanishing rate of the sequence $c_{n}$ to the vanishing rate of the $L^{2}$-norms of the data $f_{n}$ in the reinforcement sets $\Gamma^{n}$.

Proof The proof is achieved when we prove

$$
\left\|u_{n}\right\|_{L^{2}\left(Q \backslash \Gamma^{n}\right)}^{2}+\left\|\nabla u_{n}\right\|_{L^{2}\left(Q \backslash \Gamma^{n}\right)}^{2} \leqslant C
$$

where the constant $C$ does not depend on $n$. In order to show this estimate, we proceed by contradiction as in the proof of Theorem 5.3. Suppose that for every $m$ in $\mathbb{N}$, there exists an increasing sequence of indices $n_{m}$ and a sequence $u_{n_{m}}$, that we shall denote, from now on, simply by $u_{m}$ such that

$$
A_{m}:=\left(\left\|u_{m}\right\|_{L^{2}\left(Q \backslash \Gamma^{m}\right)}^{2}+\left\|\nabla u_{m}\right\|_{L^{2}\left(Q \backslash \Gamma^{m}\right)}^{2}\right)^{\frac{1}{2}} \geq m .
$$

Set

$$
v_{m}:=\frac{u_{m}}{A_{m}}
$$

we have

$$
\left\|v_{m}\right\|_{L^{2}\left(Q \backslash \Gamma^{m}\right)}^{2}+\left\|\nabla v_{m}\right\|_{L^{2}\left(Q \backslash \Gamma^{m}\right)}^{2}=1,
$$


that is,

$$
\left\|v_{m}\right\|_{H^{1}\left(Q \backslash \Gamma^{m}\right)}^{2}=1 .
$$

We denote by $v_{m, i}^{*}$ an extension of $\left.\left(v_{m}\right)\right|_{\hat{Q}_{i}^{m}}$ to $Q$ such that $v_{m, i}^{*} \in H^{1}(Q)$ and

$$
\left\|v_{m, i}^{*}\right\|_{H^{1}(Q)}^{2} \leq C
$$

with $C$ independent of $m, i=1,2$ (see Theorem 5.7 in [9]). Then, there exist a function $v_{1}^{*}$ in $H^{1}(Q)$ and a subsequence (still denoted by $v_{m, 1}^{*}$ ) that converges to $v_{1}^{*}$ weakly in $H^{1}(Q)$ (and strongly in $H^{s}(Q)$ for $0 \leq s<1$ ). Analogously there exist a function $v_{2}^{*}$ in $H^{1}(Q)$ and a subsequence (still denoted by $v_{m, 2}^{*}$ ) that converges to $v_{2}^{*}$ weakly in $H^{1}(Q)$ (and strongly in $H^{s}(Q)$ for $\left.0 \leq s<1\right)$. From (7.24) and the construction of $\left(\varphi_{1}\right)_{m}$ [see (7.14)] we deduce that $0 \in \mathcal{F}_{m}$, hence

$$
a_{m}\left(u_{m}, u_{m}\right) \leq \int_{Q} f_{m} u_{m} d x d y
$$

and

$$
\lambda\left\|\nabla v_{m}\right\|_{L^{2}\left(Q \backslash \Gamma^{m}\right)}^{2}+c_{m} \sigma_{m} \int_{\Gamma^{m}}\left|\nabla v_{m}\right|^{2} w^{m} d x d y \leq \frac{1}{A_{m}} \int_{Q} f_{m} v_{m} d x d y ;
$$

by using (7.23), (7.30), (7.47), (7.52), and Theorem 3.1 in [14], we obtain

$$
\lambda\left\|\nabla v_{m}\right\|_{L^{2}\left(Q \backslash \Gamma^{m}\right)}^{2}+\left(1-\frac{1}{A_{m}}\right) c_{m} \sigma_{m} \int_{\Gamma^{m}}\left|\nabla v_{m}\right|^{2} w^{m} d x d y \leq C \frac{1}{A_{m}} .
$$

Hence, by the weak lower semi-continuity of the norm, we obtain, for any fixed $m_{0}$,

$$
\left\|\nabla v_{i}^{*}\right\|_{L^{2}\left(\hat{Q}_{i}^{m_{0}}\right)}^{2} \leq \liminf _{m}\left\|\nabla v_{m, i}\right\|_{L^{2}\left(\hat{Q}_{i}^{m_{0}}\right)}^{2}=0
$$

then $v_{i}^{*}=k_{i}$ a. e. in $\hat{Q}_{i}^{m_{0}}$. We observe that $k_{i} \geq 0$ : in fact, by construction $v_{m} \geq$ $\frac{1}{A_{m}} \min _{\bar{Q}}\left(\varphi_{1}\right)$. As $m_{0}$ is arbitrary, we deduce that $v_{i}^{*}=k_{i}$ a.e. in $Q_{i}$ and $\nabla v_{m, i}^{*}$ weakly converges to 0 in $L^{2}\left(Q_{i}\right), i=1,2$. We show that $k_{i}=0$ : if $k_{i}>0$ we obtain a contradiction with (7.25). In fact, from (7.55), we obtain

$$
0 \leq \int_{Q} f_{m} v_{m} d x d y
$$

We have that

$$
\begin{aligned}
& \int_{Q} f_{m} v_{m} d x d y=\int_{\hat{Q}_{1}^{m}} f_{m} v_{m} d x d y+\int_{\hat{Q}_{2}^{m}} f_{m} v_{m} d x d y+\int_{\Gamma^{m}} f_{m} v_{m} d x d y \\
& =\int_{Q_{1}} f_{m} v_{m, 1}^{*} d x d y+\int_{Q_{2}} f_{m} v_{m, 2}^{*} d x d y+\int_{\Gamma^{m}} f_{m} v_{m} d x d y \\
& \quad-\sum_{j=1}^{2} \int_{\Gamma^{m} \cap Q_{j}} f_{m} v_{m, j}^{*} d x d y
\end{aligned}
$$

We estimate $\int_{\Gamma^{m}} f_{m} v_{m} d x d y$ by (7.30) and (7.56)

$$
\left\|v_{m}\right\|_{L^{2}\left(\Gamma^{m}\right)}^{2} \leq C\left(\frac{1}{A_{m} c_{m} \sigma_{m} \alpha^{m}}+\frac{1}{\sigma_{m} \alpha^{m}}\left\|v_{m, 2}^{*}\right\|_{H^{1}\left(Q_{2}\right)}^{2}\right) .
$$


By using (7.23), (7.47), and (7.54) we have that $\int_{\Gamma^{m}} f_{m} v_{m} d x d y \rightarrow 0$. On the other hand, the term $\sum_{j=1}^{2} \int_{\Gamma^{m} \cap Q_{j}} f_{m} v_{m, j}^{*} d x d y$ tends to 0 by the strong convergence of the functions $v_{m, j}^{*}$ in $L^{p}(Q), p>2$, and by (7.23). From (7.25), (7.58), and (7.59) we deduce

$$
0 \leq \lim \int_{Q} f_{m} v_{m} d x d y=k_{1} \int_{Q_{1}} f d x d y+k_{2} \int_{Q_{2}} f d x d y \leq 0
$$

and hence by (7.25) we have $k_{i}=0$. On the other hand, if $k_{i}=0$ we have a contradiction with (7.52). In fact, by (7.30), (7.52), (7.56)

$$
\begin{aligned}
1 & =\left\|v_{m}\right\|_{L^{2}\left(\hat{Q}_{1}^{m}\right)}^{2}+\left\|v_{m}\right\|_{L^{2}\left(\hat{Q}_{2}^{m}\right)}^{2}+\left\|\nabla v_{m}\right\|_{L^{2}\left(\hat{Q}_{1}^{m}\right)}^{2}+\left\|\nabla v_{m}\right\|_{L^{2}\left(\hat{Q}_{2}^{m}\right)}^{2} \\
& \leq\left\|v_{m, 1}^{*}\right\|_{L^{2}\left(Q_{1}\right)}^{2}+\left\|v_{m, 2}^{*}\right\|_{L^{2}\left(Q_{2}\right)}^{2}+C \frac{1}{A_{m} \lambda} .
\end{aligned}
$$

By taking into account (7.51) and the strong convergence of $v_{m_{k}, i}^{*}$ to zero in $L^{2}\left(Q_{i}\right)$, we obtain a contradiction. Then estimate (7.50) is proved and we can repeat the proof of Theorem 5.2 in order to show that the sequence of the solutions $u_{n}$ [defined in (7.27)] converges to the function $u$ [defined in (7.28)] weakly in $H_{l o c}^{1}\left(Q_{i}\right), i=1,2$. By proceeding as in the proof of Theorem 5.3, we can show that assumptions (7.48) and (5.21) provide the weak convergence in $L^{2}(Q)$ and assumption (5.3) provides the strong convergence in $L^{2}(Q)$.

Acknowledgments The authors wish to thank the referee for many suggestions. The work was partially supported by Grant "Sapienza" 2013. The authors are members of GNAMPA (INdAM).

\section{References}

1. Acerbi, E., Buttazzo, G.: Reinforcement problems in the calculus of variations. Ann. Inst. H. Poincaré Anal. Non Linear 3(4), 273-284 (1986)

2. Attouch, H.: Variational convergence for functions and operators. In: Pitman Advanced Publishing Program, London (1984)

3. Brezis, H., Caffarelli, L.A., Friedman, A.: Reinforcement problems for elliptic equations and variational inequalities. Ann. Mat. Pura Appl. (4) 123, 219-246 (1980)

4. Brillard, A., El Jarroudi, M.: On the interface boundary conditions between two interacting incompressible viscous fluid flows. J. Differ. Equ. 255(5), 881-904 (2013)

5. Buttazzo, G., Dal Maso, G., Mosco, U.: Asymptotic behaviour for Dirichlet problems in domains bounded by thin layers. Partial differential equations and the calculus of variations, vol. I. In: Progress in Nonlinear Differential Equations and Their Applications, vol. 1, pp. 193-249. Birkhäuser, Boston (1989)

6. Caffarelli, L.A., Friedman, A.: Asymptotic estimates for the dam problem with several layers. Indiana Univ. Math. J. 27(4), 551-580 (1978)

7. Caffarelli, L.A., Friedman, A.: Reinforcement problems in elastoplasticity. Rocky Mt. J. Math. 10, 155184 (1980)

8. Caffarelli, L.A.: The obstacle problem revisited. J. Fourier Anal. Appl. 4(4-5), 383-402 (1998)

9. Capitanelli, R.: Asymptotics for mixed Dirichlet-Robin problems in irregular domains. J. Math. Anal. Appl. 362(2), 450-459 (2010)

10. Capitanelli, R.: Robin boundary condition on scale irregular fractals. Commun. Pure Appl. Anal. 9(5), 1221-1234 (2010)

11. Capitanelli, R., Vivaldi, M.A.: Insulating layers on fractals. J. Differ. Equ. 251(4-5), 1332-1353 (2011)

12. Capitanelli, R., Vivaldi, M.A.: Trace theorems on scale irregular fractals. In: Classification and Application of Fractals, pp. 363-381. Nova Science Publishers, New York (2011)

13. Capitanelli, R., Vivaldi, M.A.: On the Laplacean transfer across fractal mixtures. Asymptot. Anal. 83(1-2), 1-33 (2013)

14. Capitanelli, R., Lancia, M.R., Vivaldi, M.A.: Insulating layers of fractal type. Differ. Integral Equ. 26(910), 1055-1076 (2013) 
15. El Jarroudi, M., Brillard, A.: Asymptotic behaviour of contact problems between two elastic materials through a fractal interface. J. Math. Pures Appl. (9) 89(5), 505-521 (2008)

16. Falconer, K.J.: The geometry of fractal sets. In: Cambridge Tracts in Mathematics (1985)

17. Jones, P.W.: Quasiconformal mapping and extendability of functions in Sobolev spaces. Acta Math. 147, 71-88 (1981)

18. Jonsson, A., Wallin, H.: Function spaces on subsets of $\mathbb{C}^{n}$. Math. Rep. 2(1), xiv+221 (1984)

19. Hutchinson, J.E.: Fractals and selfsimilarity. Indiana Univ. Math. J. 30, 713-747 (1981)

20. Kinderlehrer, D., Stampacchia, G.: An introduction to variational inequalities and their applications. Pure Appl. Math. 88 (1980)

21. Li, J., Zhang, K.: Reinforcement of the Poisson equation by a thin layer. Math. Models Methods Appl. Sci. 21(5), 1153-1192 (2011)

22. Lions, J.L., Stampacchia, G.: Variational inequalities. Commun. Pure Appl. Math. 20, 493-519 (1967)

23. Maz'ya, V.G., Poborchi, S.V.: Differentiable Functions on Bad Domains. World Scientific, River Edge (1997)

24. Mosco, U.: Convergence of convex sets and of solutions of variational inequalities. Adv. Math. 3, 510-585 (1969)

25. Mosco, U.: Composite media and asymptotic Dirichlet forms. J. Funct. Anal. 123(2), 368-421 (1994)

26. Mosco, U., Vivaldi, M.A.: An example of fractal singular homogenization. Georgian Math. J. 14(1), 169-193 (2007)

27. Mosco, U., Vivaldi, M.A.: Fractal reinforcement of elastic membranes. Arch. Ration. Mech. Anal. 194(1), 49-74 (2009)

28. Mosco, U., Vivaldi, M.A.: Vanishing viscosity for fractal sets. Discrete Contin. Dyn. Syst. 28(3), 12071235 (2010)

29. Mosco, U., Vivaldi, M.A.: Thin fractal fibers. Math. Methods Appl. Sci. 36(15), 2048-2068 (2013)

30. Sánchez-Palencia, E.: Problèmes de perturbations liés aux phénomènes de conduction à travers des couches minces de grande résistivité. J. Math. Pures Appl. (9) 53, 251-269 (1974)

31. Stampacchia, G.: Formes bilinéaires coercives sur les ensembles convexes. C. R. Accad. Sci. Paris 258, 4413-4416 (1964)

32. Troianiello, G.M.: The University Series in Mathematics. Elliptic differential equations and obstacle problems. Plenum Press, New York (1987) 